\title{
Martian moons exploration MMX: sample return mission to Phobos elucidating formation processes of habitable planets
}

\author{
Kiyoshi Kuramoto ${ }^{1,2^{*}} \mathbb{D}$, Yasuhiro Kawakatsu², Masaki Fujimoto², Akito Araya ${ }^{3}$, Maria Antonietta Barucci ${ }^{4}$, \\ Hidenori Genda ${ }^{5}$, Naru Hirata ${ }^{6}$, Hitoshi Ikeda ${ }^{7}$,Takeshi Imamura ${ }^{3}$, Jörn Helbert ${ }^{8}$, Shingo Kameda ${ }^{2,9}$, \\ Masanori Kobayashi ${ }^{10}$, Hiroki Kusano ${ }^{11}$, David J. Lawrence ${ }^{12}$, Koji Matsumoto ${ }^{13,14}$, Patrick Michel ${ }^{15}$, \\ Hideaki Miyamoto ${ }^{3}$, Tomokatsu Morota ${ }^{3}$, Hiromu Nakagawa ${ }^{16}$, Tomoki Nakamura ${ }^{16}$, Kazunori Ogawa ${ }^{17}$, \\ Hisashi Otake ${ }^{2}$, Masanobu Ozaki ${ }^{2,14}$, Sara Russell ${ }^{18}$, Sho Sasaki ${ }^{19}$, Hirotaka Sawada ${ }^{2}$, Hiroki Senshu ${ }^{10}$, \\ Shogo Tachibana ${ }^{2,3}$, Naoki Terada ${ }^{16}$, Stephan Ulamec ${ }^{20}$, Tomohiro Usui ${ }^{2}$, Koji Wada ${ }^{10}$, Sei-ichiro Watanabe ${ }^{21}$ and \\ Shoichiro Yokota ${ }^{19}$
}

\begin{abstract}
Martian moons exploration, MMX, is the new sample return mission planned by the Japan Aerospace Exploration Agency (JAXA) targeting the two Martian moons with the scheduled launch in 2024 and return to the Earth in 2029. The major scientific objectives of this mission are to determine the origin of Phobos and Deimos, to elucidate the early Solar System evolution in terms of volatile delivery across the snow line to the terrestrial planets having habitable surface environments, and to explore the evolutionary processes of both moons and Mars surface environment. To achieve these objectives, during a stay in circum-Martian space over about 3 years MMX will collect samples from Phobos along with close-up observations of this inner moon and carry out multiple flybys of Deimos to make comparative observations of this outer moon. Simultaneously, successive observations of the Martian atmosphere will also be made by utilizing the advantage of quasi-equatorial spacecraft orbits along the moons' orbits.
\end{abstract}

Keywords: Phobos, Deimos, Mars, Sample return mission, Early Solar System, Habitable planet

\footnotetext{
*Correspondence: keikei@ep.sci.hokudai.ac.jp

${ }^{1}$ Hokkaido University, Sapporo, Japan

Full list of author information is available at the end of the article
}

\section{Springer Open}

(c) The Author(s) 2021. Open Access This article is licensed under a Creative Commons Attribution 4.0 International License, which permits use, sharing, adaptation, distribution and reproduction in any medium or format, as long as you give appropriate credit to the original author(s) and the source, provide a link to the Creative Commons licence, and indicate if changes were made. The images or other third party material in this article are included in the article's Creative Commons licence, unless indicated otherwise in a credit line to the material. If material is not included in the article's Creative Commons licence and your intended use is not permitted by statutory regulation or exceeds the permitted use, you will need to obtain permission directly from the copyright holder. To view a copy of this licence, visit http://creativecommons.org/licenses/by/4.0/. 


\section{Graphical Abstract}

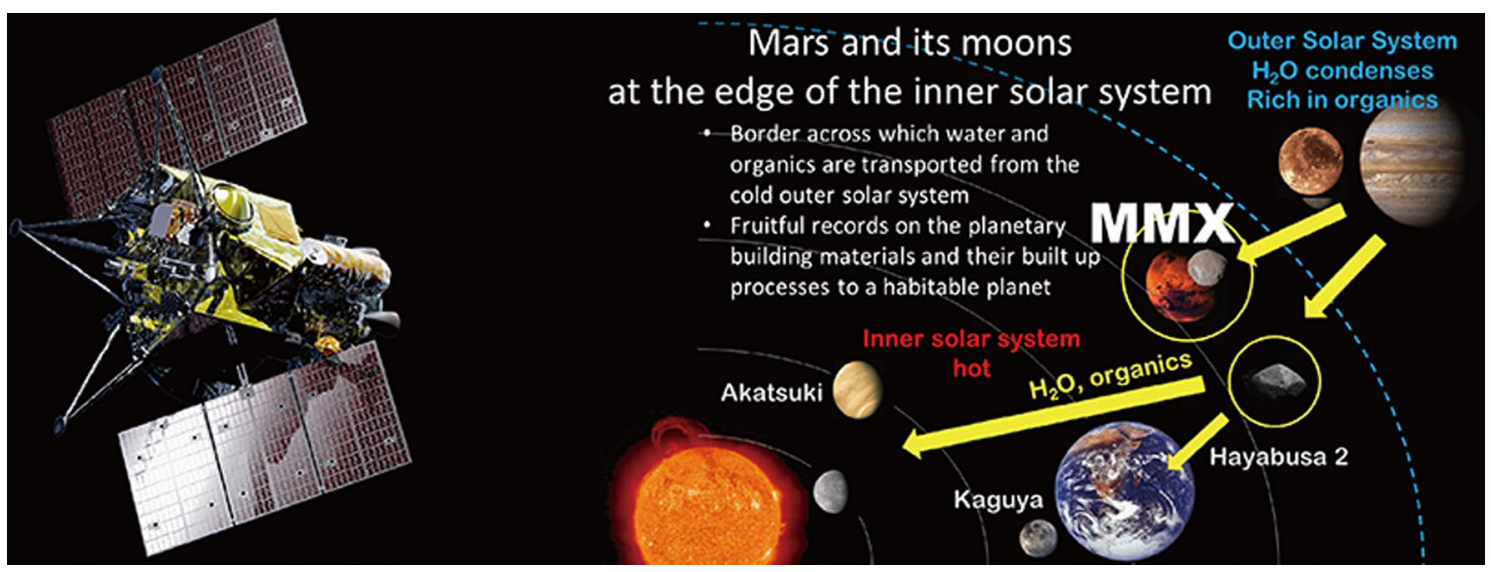

\section{Introduction}

Among the numerous moons currently known in the Solar System, there are only three orbiting terrestrial planets, Phobos and Deimos being the two of them. The exploration of the Earth's Moon, which includes sample return missions, has provided us a wealth of data revealing and constraining both its formation and evolution processes and those of the parent planet. The same is probably true for the two Martian moons which also likely formed associated with the formation of their parent planet. However, the direct exploration of the Martian moons has been quite limited so far.

The Martian Moons eXploration (MMX) mission will carry out a direct, extensive survey of the Martian moons including the first sample return from one of these moons. The past explorations of the Martian moons were largely limited to flybys by orbiters approaching Mars. The first close-up imaging of Phobos and Deimos was conducted by the Viking mission, which found their irregular shapes and their albedos as low as those of asteroids thought to have carbonaceous compositions (Veverka and Duxbury 1977; Tolson et al. 1978; Pang et al. 1978, 1980). This result favored the capture hypothesis, which had been proposed earlier for the origin of both moons but was considered dynamically difficult (e.g., Burns, 1972). Direct exploration of Phobos was attempted by Phobos 1 (1988), Phobos 2 (1988-1989), and Phobos-Grunt (2011), but they failed except for limited data acquisition by Phobos 2 in the vicinity of Phobos (Duxbury et al. 2014 for a comprehensive summary of the past explorations of Phobos and Deimos). Although several mission concepts have been proposed to explore the Martian moons, MMX is currently the only approved mission targeting the Martian moons.
For the last two decades, JAXA has been a pioneer in small-body sample return missions, accumulating unique experience through the first asteroid sample return mission Hayabusa (originally named MUSES-C, 2003-2010) to the S-type asteroid Itokawa followed by the Hayabusa2 mission (2014-present) to the C-type asteroid Ryugu. Both missions have been successfully revealing how solid materials were built up to form small bodies which then migrated to near-Earth orbits involving disruption and re-accumulation processes during Solar System evolution (e.g., Fujiwara et al. 2006; Watanabe et al. 2019; Sugita et al. 2019; Michel et al. 2020). In particular, the Hayabusa 2 mission is elucidating processes that may have supplied water and organics to Earth. MMX will expand these Japanese experiences and successes in small-body explorations to the Martian moons.

As a Mars orbiter, on the other hand, MMX will extend JAXA's experiences in large-body explorations gained by Kaguya (SELENE, 2007-2009) at the Moon and ongoing Akatsuki (Planet-C, 2010-present) at Venus. The attempted Japanese Mars orbiter Nozomi (Planet-B, 1998-2003) launched by ISAS before the institution's integration into JAXA, unfortunately, resulted in contact loss during the cruise to Mars. Hence, the MMX mission is an important milestone to expand the Japanese space program to Mars, in synergy with small-body exploration programs.

This paper presents the current design of the MMX mission with a special focus on the scientific objectives as well as the mission requirements, the system architecture, and the observation operations plan to achieve those objectives. Note that the MMX mission is under development; therefore, its details may be modified in the future. 


\section{Phobos, Deimos, and Mars: genetic and evolutionary links}

Mars, the host planet of Phobos and Deimos, is the planet that has an atmosphere-covered surface environment most analogous to that of the Earth. Abundant fluvial geomorphological features such as valley networks and outflow channels strongly imply the activity of a vast amount of liquid water on the Martian surface before 3 Gya (e.g., Carr 1996). Long-term volcanic activity leaving igneous provinces and volcanoes may significantly contribute to volatile supply from the interior to the surface (e.g., Greely 1987). Recent precise imaging spectroscopy from orbits (e.g., Bibring et al. 2006) and in situ geological surveys conducted by rovers (e.g., Grotzinger et al. 2014) accumulate evidence for the widespread existence and past activity of liquid water with compositions suitable for habitats. On the other hand, Mars preserves ancient terrains with numerous impact craters and may be the only planet for which we can precisely trace the history back to the early evolution of a hydrosphere on a rocky planet. It seems, however, difficult to approach Mars formation processes through Mars surface exploration alone because resurfacing processes significantly obscure records of the planet formation. In contrast, the airless Martian moons with sizes too small to activate long-term igneous processes likely preserve materials at their formation without severe alteration, possibly providing a window to explore key processes forming Mars.

The size of Mars, which is about half of that of Earth, and the formation time of Mars which is within several Myr since the beginning of the Solar System constrained from radioisotope studies (Dauphas and Pourmand 2011) are consistent with the typical characteristics of proto-planets predicted by planet formation theory (Kokubo and Ida 1998). Here, a proto-planet refers to the product of oligarchic growth that would have occurred in each localized feeding zone of the planetesimal population. As a result of oligarchic growth, the inner Solar System may have once contained dozens of proto-planets with typical masses from lunar to Martian ones. Their subsequent mutual collisions that occurred episodically over the time scale several $10^{7}$ years mostly after the dissipation of solar nebula gas would lead the proto-Earth and proto-Venus to accumulate their current masses (Kokubo and Ida 1998). In contrast, isotopic heterogeneities of extinct radionuclide systematics observed in Martian meteorites suggest insufficient mantle mixing of Mars, implying the lack of complete melting event possibly induced by the mutual collision of proto-planets (Debaille et al. 2007; Kleine et al. 2009). As a fossil of proto-planet, therefore, Mars may be the unique research target for revealing the processes of proto-planet formation and evolution.

Inhibition of the growth of Mars exceeding the present mass implies that the population of planetesimals in the early Solar System rapidly declined in the region beyond the current orbital radius of Mars, which might be caused by the gravitational perturbation of proto-Jupiter that experienced orbital change by mechanisms such as a large-scale, gas-driven migration (e.g., Walsh et al. 2011) or an orbital instability due to mutual gravitational interaction among the giant planets (e.g., Clement et al. 2018). These proposed mechanisms may have also played an important role in delivering water-bearing objects formed in the outer Solar System to the terrestrial planets. The exploration of Martian moons may place important constraints on such a model.

The presence of water and atmosphere is believed to be a primarily necessary condition to make a rocky planet habitable. Although the origin of water and other volatiles on the terrestrial planets remains controversial, one of the dominant hypotheses is the delivery by late accreting bodies originated in the outer Solar System (Genda 2016 for review). This is because the rocky planets are thought to be born dry if they were made from solid materials accreted in the inner solar nebula where nebular gas was too warm for water vapor to condense onto dust. In the outer solar nebula, beyond the snow line, water may condense as ice, which allows the formation of icy planetesimals. A part of them may further evolve and migrate to become rocky asteroids in the asteroid belt (e.g., Walsh et al. 2011), containing hydrated minerals generated through the chemical reactions of silicates with liquid water produced by internal heating (e.g., Fujiya et al. 2012). Observed compositions of meteorites and comets suggest that such hydrated and icy bodies are also enriched in carbon and nitrogen mainly in the form of complex organic matter (e.g., Kallemeyn and Wasson 1981; Mumma and Charnley 2011). Mars is in the best position to elucidate how such volatile-rich bodies are transported in the early Solar System because it is the terrestrial planet orbiting nearest the snow line.

Compared to the Earth's Moon, Phobos and Deimos are characterized by their smallness in size, low bulk densities, irregular shapes, and very low albedos (Table 1). They share visible to near-infrared reflectance spectra with reddening trends like those of D-type and T-type asteroids which are thought to have volatile-rich, carbonaceous compositions (Rivkin et al. 2002). Their small sizes and low bulk densities appear to be within the range of undifferentiated small bodies (e.g., Burns 1978). According to the spectroscopic classification of asteroids, the above types of asteroids are likely originated in the 
Table 1 Major properties of the Martian moons

\begin{tabular}{|c|c|c|c|}
\hline Parameters & Phobos & Deimos & Ref. \\
\hline Mass $[k g]^{a}$ & $1.0626 \pm 0.0006 \times 10^{16}$ & $1.51 \pm 0.04 \times 10^{15}$ & Jacobson (2010) \\
\hline Size $[k m]^{b}$ & $13.3 \times 11.1 \times 9.1$ & $7.5 \times 6.1 \times 5.2$ & Thomas (1989) \\
\hline Volume $\left[\mathrm{km}^{3}\right]$ & $5621 \pm 154$ & $997 \pm 49$ & Thomas (1989) \\
\hline Mean density $\left[\mathrm{g} / \mathrm{cm}^{3}\right]$ & $1.89 \pm 0.05$ & $1.51 \pm 0.07$ & Mass/Nol \\
\hline Semi-major axis [Mars radius] & 2.76 & 6.92 & $\begin{array}{l}\text { JPL Solar System } \\
\text { Dynamics, https:// } \\
\text { ssd.jpl.nasa.gov/ }\end{array}$ \\
\hline Eccentricity & 0.0151 & 0.00033 & $\begin{array}{l}\text { JPL Solar System } \\
\text { Dynamics, https:// } \\
\text { ssd.jpl.nasa.gov/ }\end{array}$ \\
\hline Inclination $\left[{ }^{\circ}\right]^{c}$ & 1.093 & 1.791 & $\begin{array}{l}\text { JPL Solar System } \\
\text { Dynamics, https:// } \\
\text { ssd.jpl.nasa.gov/ }\end{array}$ \\
\hline Geometric albedo & $0.07 \pm 0.012$ & $0.068 \pm 0.007$ & $\begin{array}{l}\text { Zellner and Capen } \\
\text { (1974), Thomas et al. } \\
\text { (1996) }\end{array}$ \\
\hline
\end{tabular}

${ }^{\mathrm{a}} \mathrm{GM}$ values are converted to mass by adopting $\mathrm{G}=6.67430 \times 10^{-11} \mathrm{~m}^{3} \mathrm{~kg}^{-1} \mathrm{~s}^{-2}$

${ }^{\mathrm{b}}$ Semiaxes of the approximated triaxial ellipsoids

' Relative to the Laplace plane

region near the Jovian orbit (DeMeo and Carry 2014). These facts naturally lead to the capture hypothesis for the origin of the Martian moons; carbonaceous primitive bodies originally formed beyond the snow line of the Solar System might have migrated inward (e.g., Walsh et al. 2011) and then become captured by Mars. If this is the case, the exploration of Martian moons will provide us clues for the delivery processes of water and other volatiles from the outer Solar System to early terrestrial planets (Table 1).

The feasibility of asteroid capture to form the Martian moons is, however, controversial. To capture a heliocentric body as a satellite, dissipation of orbital energy relative to the planet is required. Tidal dissipation alone is too weak to cause capture unless the approaching velocity to Mars was unrealistically near-zero (Ververka and Burns 1980). Models of aerodynamic capture have suggested that heliocentric bodies with sizes similar to Phobos and Deimos are possibly captured by proto-Mars embedded in the solar nebula gas (Hunten 1979; Pollack et al. 1979a). The orbit of a captured body gradually circularizes and then decays toward a collision with the planetary surface. Thus, the aerodynamic capture scenario requires some mechanism to terminate the action of aerodynamic drag, such as the loss of an extended atmosphere containing a small number of capture bodies (Hunten 1979).

On the other hand, the fact that Phobos and Deimos have low orbital inclinations and eccentricities (Table 1) has been pointed out as evidence against the capture scenario (e.g., Burns 1972; Szeto 1983). Since heliocentric bodies that encounter a planet may approach from almost random directions, the resulting orbital inclinations of captured bodies are likely also random relative to the planetary equatorial plane as seen in irregular satellites of giant planets. Tidal interactions with Mars seem too weak to reduce the orbital inclinations of captured bodies during its history given initial large inclinations (e.g., Goldreich 1965; Rosenblatt 2011 for a recent review). An alternative mechanism to reduce inclination might be a gas drag in a proto-atmosphere or circumplanetary gas envelope rotated in the same direction of the planetary rotation (Kilgore et al. 1978). It remains, however, poorly understood how such atmospheric conditions may be established and effectively work to produce moons with primordial orbits that can evolve to have current parameters.

The giant impact origin hypothesis, the other leading theory for the formation of the Martian moons, could satisfy the constraints from their orbits. Tidal interactions with Mars make Phobos orbit decline and Deimos one expand. Hence, their original orbits would be near the co-rotation radius of Mars located at a distance of $\sim 6$ Martian radii from the planetary center (Burns 1978) although low primordial eccentricity of Phobos may require recent eccentricity excitation events such as gravitational resonance due to commensurabilities among the moon's orbital period and Mars rotation rate (Yoder 1982) and/or asteroid impact(s) (Burns 1972). An oblique giant impact onto Mars may produce a debris disk from which the Martian moons would be formed at inferred original orbits (Craddock 2011; Citron et al. 2015). Recent dynamical simulations of impact ejecta 


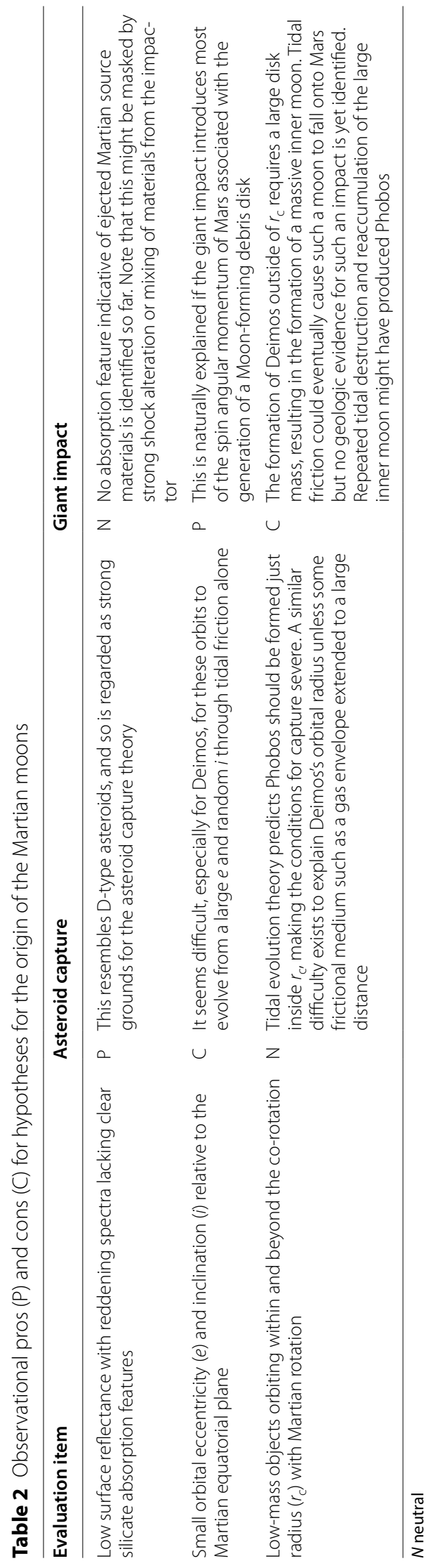


released from huge (potential) impact structures with a scale comparable to the Borealis basin, for example, succeed to explain small masses and near-circular equatorial orbits of both moons (Rosenblatt et al. 2016; Hyodo et al. 2017; Canup and Salmon 2018).

To reconcile with the formation of moons near the corotation radius, the total mass of impact ejecta extended to several Martian radii should be much larger than that of both moons, thereby leading to the formation of the inner large moon(s) through the accretion of ejecta materials (Rosenblatt et al. 2016; Hyodo et al. 2017; Canup and Salmon 2018). Such a large moon may gravitationally excite the random motion of debris in the outer ejecta disk and promote their collisional coagulation into a small number of tiny moons (Rosenblatt et al. 2016). The inner large moon(s) would eventually fall onto Mars due to tidal interactions leaving the two tiny moons with near-circular and near-equatorial orbits in the vicinity of the co-rotation radius of Mars. In an extended giant impact origin scenario, Phobos may be the youngest generation of the innermost moon that has repeated tidal break-ups, followed by orbital diffusion of disrupted debris and their partial re-accumulation during the secular tidal orbital evolution (Hesselbrock and Minton 2017). Note, however, that no clear geologic evidence has yet been identified for the mass loss of a large inner moon onto the Martian surface.

Numerical analysis of the giant impact model for the origin of the Martian moons predicts that the Martian moons consist of a mixture of impactor materials and proto-Mars materials ( $\sim 50: 50$ by Hyodo et al. 2017; 20:80 by Canup and Salmon 2018) associated with depletion of volatile elements due to impact-induced heating. Since simulated debris disks have a nearly uniform composition (e.g., Hyodo et al. 2017), these mixing ratios would apply to Phobos even if it is the latest generation of the innermost moon after the repeated cycles of tidal break-up and re-accumulation starting from an impact-generated primordial large inner moon. Therefore, the Martian moons are expected to provide us clues for the compositions of both the impactor and the protoMars, which will improve our understanding of the giant impact phenomena that resulted in the formation of both moons. On the other hand, it remains unclear how such materials lead to the current reflectance spectra of Phobos and Deimos.

Altogether, the origin of the Martian moons is still controversial to date. The current pros and cons of the two major hypotheses of the Martian moon origin are summarized in Table 2. Of course, one cannot rule out alternative scenarios such as the co-accretion of moons around a growing Mars. If MMX results lead to an alternative scenario for the origin of Martian moons, unexpected new aspects will be revealed not only for both moons, but also for the formation of Mars.

Phobos, Deimos, and their surrounding space are also expected to contain clues to understand the evolutionary processes not only on both moons, but also on Mars, such as impact flux to the Mars-moons system, resurfacing of satellite surfaces by meteoroid impacts, contamination of impact ejecta from Mars onto the moons' surfaces, ejecta transfer among the moons, the outflow of the Martian atmosphere, implantation of particles originated from solar wind and Martian atmosphere, possible gas emission from the interiors of the moons, possible formation of dust ring or torus along the moons orbits, and tidal deformation. These are valuable processes to be explored by missions to the Martian moons (e.g., Miyamoto et al. 2021). Also, from the space around the near-equatorial orbits of the Martian moons, a spacecraft can make monitoring observations of the Martian atmosphere by taking successive images and spectra covering a wide area of the planet's hemisphere. Such observations are complementary to the low altitude, close-up observations conducted by the previous Mars orbiters.

\section{Mission objectives and requirements Mission objectives}

To reveal the origin of the Martian moons and elucidate the evolution of Mars and the habitable terrestrial planets in the Solar System, the MMX mission has selected Phobos as the target for sampling and thus for detailed observations. Of course, it would be ideal to take samples from both moons, but this is infeasible because of the limitation of mission resources. The reasons for choosing Phobos as the sampling target are as follows: first, the surface of Phobos exhibits a larger spectral diversity than Deimos (Rivkin et al. 2002; Fraeman et al. 2014). This implies that the composition of Deimos may be within the range of compositional diversity of Phobos material. Note that spectral diversity might also reflect differences in physical states such as grain size distribution of surface regolith rather than compositional difference. Second, Phobos' surface likely contains a certain amount of materials ejected from young impact craters on Mars (Ramsley and Head 2013; Hyodo et al. 2019), which might provide us information on the past surface environment of Mars at the time of bedrock formation before impact cratering (Usui et al. 2020). Third, more abundant imaging data have been accumulated for Phobos, which allows us to optimize the landing strategies and operations before the launch of the spacecraft.

Referring to the advantage offered by the Martian moons for elucidating processes producing habitable terrestrial planets, the MMX mission places scientific objectives as listed in Table 3, where the major goals and 
medium objectives flow down to the specific objectives and mission requirements. The two major goals are to elucidate the origin of terrestrial planets with habitable environments and to identify and characterize important evolutionary processes of the Mars system consisting of Mars, its moons, and the circum-Martian space.

The former goal consists of two basic elements: to reveal the origins of the Martian moons and to constrain processes for planetary formation and material transport in the early Solar System. Taking into account that MMX will collect samples solely from Phobos but conduct close-up observations for both moons, this goal is broken down into three medium objectives: to identify the origin of Phobos by the combination of close-up observations and sample analyses (medium objective 1.1), to extract records of material transport processes from sample analyses $(1.2 \mathrm{a}, \mathrm{b})$ and to constrain the origin of Deimos by close-up observations (1.3).

MMX will make dense observations and sampling for Phobos. Reflecting this fact, the medium objective 1.1 is further subdivided according to the primary methods applied: close-up spectroscopic studies (MO1.1.1), sample studies (MO1.1.2) and attempts to detect possible signs of internal ice (MO1.1.3). If the composition and petrological characteristics of the Phobos bedrock material are found to be similar to those of carbonaceous meteorites generally enriched in hydrous minerals and organics, then capture origin is supported; if, on the other hand, volatile-poor, igneous characteristics are found, then the giant impact origin is supported. For close-up observations (MO1.1.1), spectroscopy characterizing the mineralogical and chemical composition of bedrock material of Phobos is important (see the "MR1.1.1" section for details). For sample analyses, petrological and chemical characterization is useful as well as isotopic measurements to achieve MO1.1.2 (see the "MR1.1.2" section). The presence of internal ice could be inferred from the detection of $\mathrm{H}_{2} \mathrm{O}$ molecules emanated from the surface (Fanale and Salvail 1990) and/or internal density heterogeneity. If a strong sign of internal ice is found, it provides strong support for the capture origin (see the "MR1.1.3" section).

Application of a series of advanced analyses for Phobos sample (see the "Sample analyses" section) is expected to provide information not only on the origin of Phobos as a Martian satellite, but also on the formation and transport of precursor bodies or the process of giant impact on primitive Mars, and the origin of colliding bodies. Either the medium objective $1.2 \mathrm{a}$ or $1.2 \mathrm{~b}$ becomes activated depending on the actual origin of Phobos. If the moons are found to be of capture origin, then the formation and dynamical transport of original bodies will be deciphered along with their cosmochemical characteristics through sample analyses (1.2a). If they are found to be of giant impact origin, then the timing and magnitude of the impact will be estimated along with the composition of the impactor, which is indicative of its formation region, by sample analyses (1.2b). For both medium objectives, determination of isotopic compositions and material dating is crucial to identify the source body type of the Phobos precursor body or the impactor and the timings of evolutionary events (see the "MR1.2a" and "MR1.2b" sections).

As for Deimos' observation (1.3), it is most important to confirm whether the genetic commonality of this moon and Phobos implied from the similar reflectance spectra at the coarse spatial resolutions is the true character or the surficial one. Due to its large distance from Mars, opportunities for close observation of Deimos by Mars exploration missions have been limited. Therefore, the coverage and spatial resolution of the Deimos spectral image data are worse than that of Phobos ones (Fraeman et al. 2012, 2014). Hence, spectral properties of bedrock materials have not yet been confirmed also for Deimos which seems largely covered with matured regolith layers (Murchie et al. 2015). Application of spectroscopy to fresh bedrock exposures expected to be associated with boulders and young impact craters is needed to achieve the medium objective 1.3 (see the "MR1.3" section).

The latter goal concerning Mars system evolution consists of three basic elements depending on the primary study targets: to reveal the evolutionary processes of both moons (2.1), to constrain the evolution of the Martian surface environment (2.2), and to understand the dynamical behaviors of Martian atmosphere regarding as an ongoing evolutionary process of the Martian surface environment (2.3).

Phobos and Deimos are similar to asteroids in size and shape, but likely suffering evolutionary processes unique for the circum-Martian environment. Since the Martian moons revolve around Mars with relatively short periods, whenever an impact occurs, a considerable fraction of ejecta grains that once escape the moons' gravity may re-impact the original moon after revolutions around Mars. Such ejecta behavior may promote the thickening of regolith layers of the moons (Ramsley and Head 2013) and cause the formation of dust rings (or tori) along the moons' orbits through impact ejecta reproduction (Soter 1971).

Phobos positioned slightly outside the tidal break-up radius of Mars may experience impact cratering events strongly influenced by the action of Martian tidal forces and/or by ejecta from Martian impact craters (e.g., Basilevsky et al. 2014). The tidally locked rotation may induce heterogeneous evolution of the surface layer between leading and trailing hemispheres due to the different 
flux and approaching velocities of impactors (Christou 2014). The ion flux from the Martian atmosphere may affect space weathering of the moons' surface along with the influx of solar wind and meteoroids (e.g., Soter 1971; Nénon et al. 2019, 2021).

To reveal impact frequency, degree of gardening, and space weathering processes, an approach combing in situ observations on impact and degradation features on both moons and dust and ion fluxes and sample analyses assessing alteration by impacts and other space weathering processes is useful. Thus, the medium objective 2.1 combines several types of studies. Its procedure will be explained in the "MR2.1" section.

The medium objective 2.2 is placed to reveal the evolution of the Martian surface environment; containing two components by the potential study of materials that came from young Martian impact craters (MO2.2.1) and observational study on the escape of the Martian atmosphere (MO2.2.2).

Materials that came from Martian impact craters are likely to be well mixed in the regolith layer of Phobos (e.g., Hyodo et al. 2019), making them difficult to distinguish by in situ observation. Thus, MO2.2.1 relies on sample analyses. Such materials likely consist of fragments of igneous, sedimentary, and metamorphic rocks (Hyodo et al. 2019), which potentially contain biosignature if Martian life exists (Hyodo and Usui 2021). Thus, they may provide unique constraints for the past Martian surface environment at the formation periods of host bedrocks of Martian craters. Potential information on past Mars obtained from such materials includes isotopic composition of surface volatile reservoirs, intrinsic magnetic field, and sedimentary environment at various geologic ages (Usui et al. 2020). It is to be noted that the availability of materials applicable to such in-depth analysis is not certain, and therefore MO2.2.1 is achievable if good samples are obtained (see the "MR2.2.1" section).

The other approach to understanding the evolution of the Martian surface environment is the aeronomy study to constrain the atmospheric escape from Mars (MO2.2.2). Atmospheric escape is one of the significant ongoing processes at Mars that have driven the evolution of the Martian surface environment over a geologic time scale (e.g., Jakosky et al. 1994). The isotopic fractionation of escaping species changes the isotopic ratios of residue volatile elements over geological time. Thus, the isotopic fractionation factor at escape is an important parameter to interpret the isotopic difference among Martian volatile reservoirs in terms of the time scale and magnitude of atmospheric loss. Past Martian missions and ground-based observations have progressively revealed the isotopic composition of volatile elements in the Martian atmosphere and sedimentary materials along with analyses of Martian meteorites (e.g., Leshin et al. 2013; Mahaffy et al. 2013; Usui et al. 2015; Villanueva et al. 2015; Jakosky et al. 2018). However, isotopic fractionation of escaping elements, which probably depends on the level of solar activity, has been poorly constrained by previous studies especially for ion species such as $\mathrm{O}^{+}$and $\mathrm{C}^{+}$due to the lack of reliable observation of escaping species and difficulty in theoretical modeling (Chassefière and Leblanc 2004). To give an estimate for the escaped amount atmosphere by new observational determination of the fractionation factor is the primary component of MO2.2.2 (see the "MR2.2.2" section).

The medium objective 2.3 reflects an advantage in observations of the Martian atmosphere from orbits near the moons as mentioned previously. The Martian atmosphere exhibits various meteorological activities. Among them, atmospheric transport of water vapor and dust plays a particularly important role in the evolution of the Martian climate system. The lateral water transport is an elementary process for global-scale redistribution of water storage among surface reservoirs over the time scale of changes in obliquity and orbital parameters such as eccentricity and the argument of periapsis caused by gravitational perturbations by other planets (e.g., Mustard et al. 2001). Dust that distributes everywhere in the Martian atmosphere with variable content significantly influences the radiative budget of the thin atmosphere since dust is the primary absorber for visible and infrared radiation (Pollack et al. 1979b). Therefore, dust strongly affects the atmospheric thermal budget and dynamics, which in turn influence water vapor transport, including one across the vertical direction reaching the upper atmosphere where photolysis of water molecules takes place associated with the subsequent escape to space (e.g., Heavens et al. 2018).

However, it remains poorly constrained how the dust transport is coupled with atmospheric dynamics and water transport due to the paucity of continuous imaging observations that can capture developments of dust events (Ogohara et al. 2021). To reveal coupled dynamics of dust and water vapor transport by new atmospheric monitoring is the primary component of the medium objective 2.3 (see the "MR2.3" section).

Considering that the MMX mission has been assembled from the primary objective of exploring the Martian moons, the mission objectives are prioritized as shown by the marks in Table 3. Science objectives related to the origin and evolution of Phobos, which are certainly achievable if the mission proceeds correctly, are given the top priority; science objectives that depend on results of observation and sample analysis are given the second priority; and science objectives related to the surface 
evolution of Mars are given the third priority, as shown in the following.

Determination of the origin of Phobos by spectroscopic observations (MO1.1.1) and by sample analyses (MO1.1.2) has the highest priority as well as the identification of weathering and evolutionary processes of Phobos surface (MO2.1.1). Constraining Phobos' origin from its molecular release rate and mass distribution (MO1.1.3) is given high priority as well as revealing the surface distribution of constituent materials in Deimos in comparison with those of Phobos to constrain the origin of Deimos (MO1.3.1). The second priority is also given for constraining the initial conditions of early Solar System processes such as planetary migration through clarification of the formation of primitive materials and bodies and the capture process (MO1.2a.1) and estimating the magnitude and timing of the giant impact along with placing constraints for planetary migration, material transport, and planetary formation processes (MO1.2b.1). The achievability of these two objectives depends on the origin of Phobos to be determined. Objectives related to the observations of the Martian atmosphere (MO2.2.2 and MO2.3.1) and the potential study of grains ejected from young impact craters (MO2.2.1) are given third priority.

\section{Mission requirements}

Corresponding to each mission objective, the mission requirements are deduced by reflecting the current knowledge of the Martian moons and their parent planet (Table 3). The general scientific grounds for each mission requirement are described below.

MR1.1.1 to constrain Phobos origin by in situ observations MR1.1.1 summarizes the requirements for remote sensing observations to constrain the origin of Phobos. Previously obtained visible and near-infrared reflectance spectra of Phobos are almost featureless with possible weak features indicative of olivine and pyroxene (Gendrin et al. 2005) and phyllosilicates (Fraemann et al. 2014), but there remain the limitation of $\mathrm{S} / \mathrm{N}$, spatial resolution, and/or coverage. ISM (infrared spectrometer) on board Phobos 2 spacecraft obtained Phobos spectra over a quarter Phobos hemisphere by 24 times 25 pixels in $0.8-$ $1.5 \mu \mathrm{m}$ and $1.6-3.1 \mu \mathrm{m}$ wavelength ranges with $\mathrm{S} / \mathrm{N}$ from 10 to 300 dependent on wavelength (Gendrin et al. 2005). Current best spectroscopic data may be those obtained by CRISM aboard the Mars Reconnaissance Orbiter (Fraeman et al. 2012, 2014), which has the spatial resolutions $120-350 \mathrm{~m} / \mathrm{px}$ for the sub-Mars hemisphere of Phobos in $0.4-3.1 \mu \mathrm{m}$ range though lacking reliable data around $2.7 \mu \mathrm{m}$ due to the boundary of instrument filters. Spectral feature around $2.7 \mu \mathrm{m}$ is important because it includes characteristic absorptions from hydroxyl and water molecules. If hydrated minerals are found in fresh bedrock exposures, this provides strong evidence for the capture origin of Phobos.

Since the Phobos surface is possibly affected by space weathering and late accreted materials, it is valuable to determine reflectance spectra for fresh materials exposed around impact craters associated with bright ejecta and on boulders identified on Phobos (e.g., Basilevsky et al. 2014). To achieve such observations, a spatial resolution of $20 \mathrm{~m}$ or better is required for spectroscopic mapping of major areas on Phobos (see the "MR2.1" section for details). Spectroscopic imaging with a spatial resolution of $1 \mathrm{~m}$ or better is necessary for $\sim 50 \mathrm{~m}$ area including sampling site to characterize the geologic context of sampling sites. Requirements for visual and infrared spectral measurements will be summarized in the later "Requirements for spectroscopic imaging" section combining other requirements to observe the geology of both moons and the Martian atmosphere.

The mean $\mathrm{Si} / \mathrm{Fe}$ ratio on the hemisphere scale, which is observable by a gamma-ray spectrometer and potentially by an ion mass spectrum analyzer, also provides an important clue to revealing Phobos origin. If capture origin is the case, $\mathrm{Si} / \mathrm{Fe}$ ratio is expected to be within the range of chondritic meteorites. If the giant impact origin is the case, $\mathrm{Si} / \mathrm{Fe}$ ratio may be $>20 \%$ larger than the chondritic range (1.1-2.0 Wasson and Kallemeyn 1988) due to the contribution of Mars mantle materials (2.9-3.7 Yoshizaki and McDonough 2020 and references therein). Therefore, the determination of $\mathrm{Si} / \mathrm{Fe}$ ratio with precision less than $20 \%$ for global Phobos is the requirement for elemental abundance measurements. If possible, the determinations of $\mathrm{K}$ concentration with chondritic range (300-800 ppm) and hydrogen concentration above $100 \mathrm{ppm}$ (typical concentration for relatively $\mathrm{H}_{2} \mathrm{O}$ poor carbonaceous chondrites, Wasson and Kallemeyn 1988) with precision less than 50\% for global Phobos also contribute to assessing whether Phobos is capture origin or giant impact origin. If the elemental measurement is performed by gamma-ray and neutron spectrometry, it needs integration of photons originated from Phobos from low altitude less than about one Phobos mean radius to reduce the background noise (Lawrence et al. 2019).

\section{MR1.1.2 to determine Phobos origin from sample}

MR1.1.2 describes the mission requirement for sampling and sample analyses to determine Phobos' origin. To collect Phobos indigenous materials from the surface possibly contaminated by exogenic materials, a large amount of granular sample is necessary for statistical sorting by the isotopic composition. The average mixing ratio of 
external materials is estimated to be about $0.1 \%$ as likely (Hyodo et al. 2019) and several \% at most (Ramsley and Head 2013). The distribution of external material in the surface regolith layer is expected to be almost homogeneous due to gardening processes and randomness of pre-impact trajectories (Ramsley and Head 2013; Hyodo et al. 2019). On the other hand, there is a possibility that Phobos indigenous materials have compositional diversity. This is implied from the observed regional difference in reflectance spectra represented by the red unit and blue one (Rivkin et al. 2002; Fraeman et al. 2014). Here the blue unit stands for areas having weaker reddening trends. Note that it could represent differences in the degree of space weathering, alternatively (e.g., Basilevsky et al. 2014).

Due to the current lack of direct measurement, the grain size distribution of Phobos regolith remains uncertain. According to the interpretation of Phobos thermal inertia from the modeling of thermal conduction through granular layers, the typical grain size in Phobos regolith is estimated to be $\sim 1 \mathrm{~mm}$ (Gundlach and Blum 2013). An expanded method incorporating the physical relationships between porosity and grain size under the action of intra-grain cohesion forces and Phobos's gravity suggests the typical size of $\sim 2 \mathrm{~mm}$ (Kiuchi and Nakamura 2014). Among ejecta released from the Phobos surface by impacts, grains smaller than $\sim 300 \mu \mathrm{m}$ would be preferentially lost to the parent planet or outer space due to the action of solar radiation pressure (Ramsley and Head 2013) while large grains may return onto the surface of Phobos after areocentric motion. For those reasons, the regolith grains are likely to have sizes mainly between $\sim 300 \mu \mathrm{m}$ and $\sim 2 \mathrm{~mm}$.

Application of textural, mineralogical, and chemical analyses for grains is useful to judge the origin of Phobos. The estimated size range of Phobos sample grains allows us these analyses in detail as demonstrated for Itokawa dust particles with much smaller sizes (e.g., Nakamura et al. 2011). If the majority of Phobos samples are petrologically like chondritic materials, this strongly supports the capture origin of Phobos. On the other hand, if the samples are dominated by materials with textures and compositions reflecting high-temperature melting and partial vaporization, this supports giant impact origin.

Recent improvements in the accuracy of isotope measurements have revealed that meteorite groups and planetary materials have different combinations of isotopic anomalies (Warren 2011). It has long been known that there are differences in isotopic anomalies among meteorite groups (Clayton 1993); when $\mathrm{Cr}$ isotopic ratio anomalies are included, almost all known meteorite groups and planetary materials can be distinguished (Warren 2011). Therefore, from the isotope ratio analysis of the sample, it is possible to determine the source body type of Phobos.

Measurement of ${ }^{53} \mathrm{Cr} /{ }^{54} \mathrm{Cr}$ ratio with a precision of $10^{-6}$ enough to resolve source body type (Trinquier et al. 2008) requires a sample grain of a few $\mathrm{mg}$ or size $\sim 1 \mathrm{~mm}$ if the $\mathrm{Cr}$ concentration is $\sim 3000 \mathrm{ppm}$ equivalent to carbonaceous chondrites (Fujiya et al. 2021). Here, a silicate grain with a density of $3 \mathrm{~g} / \mathrm{cm}^{3}$ is supposed. For Itokawa samples with tens $\mu \mathrm{m}$, oxygen isotope analysis was achieved with precision sufficient to resolve source body type (e.g., Yamamoto et al. 2011; Yurimoto et al. 2011; Nakashima et al. 2013). Other isotope systems such as ${ }^{50} \mathrm{Ti} /{ }^{47} \mathrm{Ti}$ are also valuable (Warren 2011) and measurable with sufficient precision for $\sim 1 \mathrm{~mm}$ grains (Fujiya et al. 2021). The majority of Phobos sample grains are expected to concentrate in a certain compositional range which shows the Phobos indigenous material and source body type.

The collection of $\geq 10$ g samples is required to identify the composition of Phobos indigenous materials for the following reasons. Samples with a total mass of $10 \mathrm{~g}$ are expected to contain an order of $10^{3}-10^{5}$ grains, assuming $3 \mathrm{~g} / \mathrm{cm}^{3}$ as the typical grain density. In this case, $100-10^{4}$ grains can be secured in $1 / 10$ of the sample to be used for the initial analysis after screening. This volume of sample grains allows us to identify the principal component of sample materials concerning their chemical and isotopic compositions. The obtained principal component will be concluded to be Phobos indigenous materials through the confirmation of its consistency with the Phobos bedrock composition constrained by in situ observations. Considering the possible large-scale heterogeneity in the material composition on Phobos, the sampling sites are desired to be selected from the red unit and blue unit regions, respectively.

To obtain materials minimally affected by space weathering caused by solar wind and meteoroid fluxes, samples should include materials from a depth of $>2 \mathrm{~cm}$ that is deeper than the penetration depth of solar wind (Nishiizumi et al. 2009). Also, to guarantee the collected samples to be Phobos indigenous materials, material distribution on the almost entire Phobos as well as the geologic context of sampling sites should be constrained by imaging and spectroscopic mapping. Requirements for these observations reflect the known geological properties of Phobos, which will be described later in the "MR2.1" section that describes requirements to observations for precise Phobos geology.

\section{MR1.1.3 to constrain Phobos internal structure}

Mission requirements for the acquisition of data related to Phobos's internal structure that independently provide constraints on Phobos origin are summarized in MR1.1.3. The low density of Phobos compared to intact 
rocks possibly reflects the existence of water ice in the deep interior or a significant porosity in this moon at all depths. According to the thermal evolution model of Phobos (Fanale and Salvail 1990), the emanation of water molecules may continue at present depending on the thermal and molecular conductivities and assuming that Phobos was originally a primitive small body bearing water ice. $10^{22}-10^{23}$ molecules/s is a possible emanation rate at present (Fanale and Salvail 1990). Thus the observation that can detect this amount of emission is required. This is possible using in situ ion mass spectrometry (Yokota et al. 2021). The emanation of $\mathrm{H}_{2} \mathrm{O}$ molecules, if detected, will strongly support the capture of an ice-bearing small body for Phobos' origin. Requirements for in situ ion mass spectrometry will be summarized in the later subsection combining the observations of solar wind components, sputtered ions from Phobos, and escaping ions from the Martian atmosphere.

The icy Phobos model also implies a combination of a dense core made of a silicate-ice mixture and a porous silicate mantle under the evaporative loss of ice from the surface. If mass concentration toward the center is found from geodetic observation, it places important constraints on such a model. To make investigations to detect such density inhomogeneity is another mission requirement. The key parameters to constrain the radial distribution of mass within the interior are moments of inertia (MOI) that can be derived from short-period longitudinal libration amplitude $\theta$ and degree- 2 gravity coefficients $C_{20}$ and $C_{22}$. Improvement of the accuracy of $\theta$, $\mathrm{C}_{20}$ and $\mathrm{C}_{22}$ within $2-3 \%$ and the deduced moments of inertia enable us to constrain the central concentration of ice with $10 \%$ of total Phobos mass (Matsumoto and Ikeda 2016; Matsumoto et al. 2021). Degree-1 gravity coefficients provide the position of the center of mass relative to the geometrical center of figure that would also imply density heterogeneity reflecting inhomogeneous ice distribution. Note that density heterogeneity could also be caused by other mechanisms such as porosity change and the coalescence of planetesimals with different compositions.

Currently, the errors of gravity coefficients are too large to deduce reliable moment of inertia to constrain density inhomogeneity (Matsumoto et al. 2021). A long stay around Phobos may significantly reduce this error through the gravity field determination based on precise analysis of inertial spacecraft motion around Phobos. The detailed requirements for spacecraft orbit plan as well as observation plans to obtain precise geodetic parameters are under study.

\section{MR1.2a to understand early Solar System processes}

MR1.2a summarizes the mission requirements mainly for sample analysis when capture origin of Phobos is proven to be the case. In this case, the sample may be abundant in grains composed of unequilibrated minerals enriched in volatile elements. Chemical, mineralogical and isotopic compositions are required to identify the source body type of the Phobos precursor. Their comparison with primitive meteorites will tell us the genetic relationships of Phobos precursor and parent bodies of primitive meteorite groups in the early Solar System. Comparison with samples returned by Hayabusa, Hayabusa2, and OSIRIS-REx together with the close-up observation data of the respective asteroids is also crucial. Using the corresponding relationships among the spectroscopic taxonomy of asteroids and meteorite groups (DeMeo and Carry 2014), the source region of the Phobos precursor may be estimated. Reflectance spectra of returned samples that contain less altered materials are necessary to provide better constraints on the source asteroid type of the Phobos precursor. Volatile abundances in Phobos indigenous materials are also necessary to evaluate how Phobos-like bodies may contribute to built-up an ancient atmosphere-ocean-cryosphere system on proto-Mars.

The application of chronological analyses is required for the estimation of time and environment of the formation of the precursor body as well as for constraining the impact history during the migration in the early Solar System. To approach the chronology of the formation of primordial materials, dating with a time resolution of the order of $0.1 \mathrm{Myr}$ is required. To achieve such a time resolution, ${ }^{26} \mathrm{Al}-{ }^{26} \mathrm{Mg}$ dating may be the primary method to reveal the history of refractory material formation in the solar nebula, and ${ }^{53} \mathrm{Mn}-{ }^{53} \mathrm{Cr}$ dating may reveal thermal and aqueous alteration history on the precursor body of Phobos. Both methods that use extinct radionuclides for model age determination require refractory grain and altered grain with $10 \mu \mathrm{m}$, respectively (e.g., Kita et al. 2013; Fujiya et al. 2012), which are probably available if Phobos is similar to the carbonaceous chondrites in its composition. A high-precision absolute age determination using $\mathrm{U}-\mathrm{Pb}$ systematics requires a refractory grain of several $\mathrm{mm}$ in size (Fujiya et al. 2021); the availability of such a large grain might be limited considering the expected typical grain size.

Adding to the precise impact crater statistics that will be revealed by MMX close-up observations, the distribution of shock alteration ages of grains will provide constraints on the major impact history of Phobos including the stage of its precursor body with heliocentric orbit if capture origin is the case. At low gravity Phobos, most of the ejecta released by a small-body collision experience low shock pressures (Asphaug and Melosh 1993). In 
addition, the empirical relationship between shock pressure and shock alteration (Stöffler et al. 1991) predicts that the fraction of regolith material that undergoes shock alteration such as Ar loss is likely to be limited. Considering that the regolith is well stirred by gardening, the samples are likely to be a mixture of materials that have undergone shock alteration at various ages in the past. For Itokawa grains, shock alteration ages of $1.3 \pm 0.3 \mathrm{Ga}$ (3-grain analysis with the total mass of $\sim 3 \mu$ g equivalent to a $\sim 100 \mu \mathrm{m}$ grain; Park et al. 2015) and 2.3 $\pm 0.1 \mathrm{Ga}$ (analysis of single grain with the size of $91 \mu \mathrm{m}$; Jourdan et al. 2017) have been reported using ${ }^{39} \mathrm{Ar}-{ }^{40} \mathrm{Ar}$ dating. This indicates that determination of shock alteration age is likely to be possible with sufficient time resolution for Phobos samples expected to have larger typical grain sizes.

By obtaining the distribution of shock alteration ages, the evolution of the impact frequency of small bodies on Phobos can be estimated from the precursor body stage to the present. Considering the orbital evolution from the asteroid belt, the flux of small bodies to Phobos should have declined significantly after the capture by Mars (Schmedemann et al. 2014). If a sharp decrease in the distribution of shock alteration ages is identified with a certain age boundary, it gives an estimate for the timing of capture. A large portion of shock-altered grains might arise from the formation of voluminous impact craters such as Stickney, which may lead to peaks in the distribution of shock alteration ages. Interpretation of the shock age distribution should take into account the precise geology accessing resurfacing history around sampling sites.

Components enriched in volatile elements such as hydrous minerals, carbonates, and organic matters may also tell us material formation history and environment in the early Solar System. D/H ratio in hydrous minerals and organics and $\mathrm{N} / \mathrm{C}$ and $\mathrm{O} / \mathrm{C}$ ratios in organic matters are useful as a proxy for estimating their formation conditions. For instance, cometary materials, likely formed in the early outer Solar System and/or a parent molecular cloud, tend to exhibit high $\mathrm{D} / \mathrm{H}, \mathrm{N} / \mathrm{C}$, and $\mathrm{O} / \mathrm{C}$ ratios compared to chondritic materials (e.g., Altwegg et al. 2015; Yabuta et al. 2017). For hydrous minerals with a size $10 \mu \mathrm{m}$ or smaller, $\mathrm{D} / \mathrm{H}$ measurement is possible with $1 \%$ precision that is sufficient to distinguish between cometary and chondritic values (e.g., Altwegg et al. 2015). N/C and $\mathrm{O} / \mathrm{C}$ measurements with sufficient precision have been done for micrometeoritic organic materials with sizes several to about $10 \mu \mathrm{m}$ (e.g., Yabuta et al. 2017). These analyses may also be useful for Phobos samples expected to have larger typical grain sizes.

\section{MR1.2b to understand the moons' forming giant impact} If giant impact origin is the case, determination of textures, chemical, mineralogical and isotopic compositions, and alteration ages of Phobos indigenous materials are necessary for the estimation of the timing and magnitude of the impact event, the chemical type of the impactor, as well as the mixing ratio of materials derived from the impactor and proto-Martian mantle.

Such an impact event could occur with an impact velocity not much larger than the escape velocity of Mars, and materials ejected into areocentric orbits may inefficiently be mixed in grain-scale due to rapid solidification by efficient radiative cooling (Hyodo et al. 2017). In this case, Phobos indigenous materials may show isotopic and chemical compositions distributed on a mixing line connecting the compositions of the proto-Martian mantle and the impactor. Since the isotopic compositions of elements such as oxygen are already known for Mars from the analyses of Martian meteorites (e.g., Warren 2011), the direction of the anti-Mars end of the mixing line points to the impactor isotopic composition. From this analysis, the source body type of the impactor may be estimated. Materials that fall on isotopic end-members could also tell us the chemical composition of the proto-Martian mantle and the impactor. The range of compositional dispersion depends on the degree of material mixing and homogenization, which reflects the magnitude of the moonforming impact.

The moons' forming giant impact likely causes loss of volatile elements like $\mathrm{H}, \mathrm{Pb}, \mathrm{C}, \mathrm{Na}, \mathrm{K}$, and $\mathrm{Rb}$ from ejecta originated from Mars and the impactor due to impact heating (Hyodo et al. 2018). The degree of volatile loss can be evaluated from the comparison of volatile-element abundance of Phobos samples with those estimated for the silicate Mars (e.g., Yoshizaki and McDonough 2020) and the source body type of impactor. This would provide another constraint for the heating magnitude of the giant impact.

Note that tidal break-up and reaccumulation postulated for the scenario of repeated formation of innermost moon likely induce only small temperature rise due to low gravitational energy to bound the moons' materials. Thus, Phobos materials would experience little alteration through the reformation cycles. An effect of reformation possibly recorded in the Phobos sample might be a low degree of cosmic-ray exposure because tidal break-up may dredge up materials once existed in deep. Further study is needed on how the Phobos sample can constrain the repeated formation scenario of the innermost moon.

The timing of giant impact can be constrained by the measurement of crystallization age of returned samples. 
$\mathrm{U}-\mathrm{Pb}$ dating for phosphate phase in an Itokawa grain with the size several tens $\mu \mathrm{m}$ obtained $4.6 \pm 0.18 \mathrm{Ga}$ for the grain formation age (Terada et al. 2018). Given the range of expected grain sizes, the same dating method can be applied to Phobos samples with similar or better precision which distinguishes between the period overwrapping the main period of Mars accretion likely the first several Myr of the Solar System (Dauphas and Pourmand 2011) and that of late heavy bombardment possibly lasting to about $3.8 \mathrm{Ga}$ (e.g., Hartmann et al. 2000). An ${ }^{87} \mathrm{Rb}-{ }^{87} \mathrm{Sr}$ model age can be also measured for the timing of the impact associated with volatile loss and fractionation between Rb and Sr (Amsellem et al. 2020).

\section{MR1.3 to constrain Deimos's origin}

Mission requirements for constraining the origin of Deimos are described here. According to Viking's flyby observations, boulders as large as 150-200 m have been found on the Deimos surface (Thomas 1979). To spectroscopically characterize the bedrock material exposed on boulder surfaces, it is necessary to make spectroscopic observations at a horizontal spatial resolution of $100 \mathrm{~m}$ or less in the same wavelength range and $\mathrm{S} / \mathrm{N}$ for Phobos observation and to obtain geomorphological data at a horizontal spatial resolution of $10 \mathrm{~m}$ or less. Based on previous observations, the Deimos surface has an almost homogeneous color, while there are red and blue regions on Phobos. Therefore, Deimo's observations may be meaningful enough, even if only for a few major areas.

Deimos is known to have a south pole basin, which may be the largest impact crater on this moon comparable to Stickney on Phobos. However, the topography of this basin has been poorly determined so far due to the lack of image at good viewing conditions (Thomas 1993). A detailed study of the topography of this basin is necessary for the estimation of the Deimos's volume and thus density as well as for the geological comparison of Deimos with Phobos.

\section{MR2.1 to reveal the evolution of Phobos}

The mission requirements given by MR2.1 are specified for clarifying the surface evolution and its processes of Phobos, paying attention to the difference in boundary conditions from heliocentric small bodies as described previously. To unravel those complex processes, it is necessary to conduct observations of dust and ion fluxes in circum-Martian space, geologic mapping for precise crater chronology, global boulder distributions, and major terrains and geological features, such as grooves, and sample analyses characterizing space weathering processes.

The first information needed to understand the degree of space weathering that likely varies by location is the reflectance spectrum of fresh bedrock exposures. On Phobos, signatures of fresh bedrock exposure have been found in ejecta around fresh impact craters of tens of meters scale (Basilevsky et al. 2014) and boulders of tens of meters size (Thomas 1979; Basilevsky et al. 2014). Comparing the reflectance spectra of these regions with others, the extent and distribution of space weathering on the Phobos surface can be constrained. According to size distributions of boulders in the region eastern outside of Stickney crater (Thomas 1979; Karachevtseva et al. 2014), there are at least 100,10, and 3 boulders with diameters greater than 10,20 , and $30 \mathrm{~m}$, respectively. Therefore, to measure the reflection spectrum of fresh bedrock exposures at sufficiently many sites for a statistical survey, it is necessary to observe boulders with a diameter of at least $20 \mathrm{~m}$. This requires geomorphological imaging with resolution less than $4 \mathrm{~m}$ or less and spectroscopy with a spatial resolution of $20 \mathrm{~m}$ or less.

Grooves (100-200 m wide and 10-20 m depth linear depressions) are one of the most prominent geomorphological structures in Phobos. The grooves have been interpreted as secondary crater chains due to impact(s) on Phobos or Mars, or faults created by tidal forces or a huge impact shock on Phobos (e.g., Murrey et al. 1994; Murray and Heggie 2014). A particularly important piece of information in constraining the formation is to distinguish between chain craters of impact origin and sinkholes into the inner cavity. The above-mentioned high-resolution imaging, mainly of the walls and the surrounding area, are useful to assess the continuity of the groove topography, the presence of impact pit and ejecta, and the presence of faults.

On Eros, an asteroid with a size similar to Phobos, the number density of impact craters less than $1 \mathrm{~km}$ in diameter is in an equilibrium state balancing creation and disappearance, and the change in the slope of the size distribution at smaller diameters suggests that different degradation processes dominate in each size range (Richardson et al. 2005). A similar equilibrium state has been observed for Itokawa (Hirata et al. 2009). If the processes of re-impact of dust ejected from Phobos occurs continuously, the number density of small impact craters on Phobos is expected to be lower than those on Eros and Itokawa because crater degradation processes become stronger. To observe this, it is necessary to understand the density of impact craters with diameters less than $100 \mathrm{~m}$, which requires global geological mapping with spatial resolution less than $10 \mathrm{~m}$.

As for the proposed dust rings or tori along the orbits of the Martian moons (Soter 1971), a theoretically expected dust number density is $7 \times 10^{3} \mathrm{~km}^{-3}$ for dust larger than $30 \mu \mathrm{m}$ in the Phobos dust ring and several times denser in the Deimos dust ring (Krivov and Hamilton 1997). To 
constrain the dust environment around both moons, it is necessary to detect ring dust with size range and density above as well as micrometeorite flux with an expected value of about $10^{-6} \mathrm{~m}^{-2} \mathrm{~s}^{-1}$ (Grün et al 1985).

Analyses of sample grains focusing on alteration processes due to fluxes of external materials would reveal how Phobos surface, probably poor in the endogenic activity that affects material properties, has evolved in the circum-Martian environment. A histogram of shock alteration ages of sample grains may provide information on the history of high-speed impacts onto Phobos along the lines of similar studies for the Earth's Moon (e.g., Fernandes et al. 2013). Concentrations of implanted solar wind ions in weathered samples, as well as cosmic-ray exposure age, may be used to estimate the resurfacing time scale of Phobos (e.g., Nagao et al. 2011 for Itokawa sample). The concentration of exotic materials would include information on the ejecta and ion transport from Mars.

\section{MR2.2.1 to constrain Mars evolution from Phobos sample}

The mission requirements for the search for materials transferred from Mars after Phobos formation in the returned sample are given by MR2.2.1. Based on the young impact crater population on Mars and numerical simulations of impact processes, it is estimated that Phobos regolith may contain on average at least $340 \mathrm{ppm}$ of ejecta materials from Mars due to impacts during the last 500 Myr (Hyodo et al. 2019). $10 \mathrm{~g}$ of granular Phobos sample may contain several tens grains of such Martian ejecta assuming that $300 \mu \mathrm{m}$ grains, the smallest estimate of dominant grain size on Phobos (see the "MR1.1.2" section), dominate the sample mass. The larger the dominant grain size, the smaller the expected total number of young grains originated from Mars. Therefore, at least $10 \mathrm{~g}$ of sample is required to raise the expected total number to $\mathrm{O}(1)$ or higher.

Different from the giant impact case, most ejecta materials are not expected to have undergone strong shock alteration such as melting due to scale effects (Hyodo et al. 2019). If Phobos is a captured primordial undifferentiated body, ejecta from Martian impact craters will have petrological properties distinctly different from the primitive materials. Even if Phobos is of giant impact origin, materials from young Martian impact craters can be distinguished from Phobos indigenous material originated from quenched ejecta generated by the giant impact. The reliability of the identification results can be assured by comparison with the $\mathrm{O}, \mathrm{Cr}$, and $\mathrm{Ti}$ isotopic compositions in Martian meteorites (e.g., Warren 2011) and mineralogical composition data obtained from Martian meteorites and Martian explorations (e.g., McSween 2015).

If fragments of younger impact ejecta from Mars are identified in the sample grains, they would provide information on the state of the surface environment on Mars at the timing of bedrock formation at impact site(s). Their mineral assemblages, isotopic compositions, and magnetization are potential measurable proxies for the past surface environment of Mars. To make them even more valuable, the age determination of corresponding sample grains is quite important. Our capability to do this depends on grain size and mineral composition. If such measurements are successful for ejecta grains of various ages, it would significantly improve our understandings of the evolution of Mars (Usui et al. 2020).

\section{MR2.2.2 to constrain the atmospheric escape from Mars}

The mission requirement given by MR2.2.2 is placed to conduct flux measurements of major escaping ion species such as $\mathrm{O}^{+}$and $\mathrm{C}^{+}$with discriminating isotopes to improve constraints on the amount of the past atmosphere. If the fractionation factors of main atmospheric species can be estimated with a precision of less than $50 \%$, the amount of atmosphere lost to space over the past billion years can be constrained within a factor of $\sim 2$ given the known isotopic differences between the present atmosphere and sediments that trap the past isotopic composition of the atmosphere (Yokota et al. 2021). To take observational constraints for the fractionation factors, it is required to measure composition ratios and isotopic ratios of the main components of ions escaping from the current Martian atmosphere with an accuracy of $50 \%$ or less.

To measure the isotopic fractionation factors of the major components of escaping ions $\left(\mathrm{O}^{+}\right.$and $\left.\mathrm{C}^{+}\right)$with such accuracy, the isotopic flux ratios of each element are needed to be measured with the same accuracy in the induced magnetosphere of Mars. Also, solar wind parameters need to be measured outside the induced magnetosphere because the ion escape is affected by the solar wind (e.g., Brain et al. 2010). Requirements for in situ ion mass spectrometry will be summarized in the later subsection combining those for the other targets.

\section{MR2.3 to understand dust and water transport in the Martian atmosphere}

The mission requirement given by MR2.3 is placed to realize monitoring the transport of water and dust, both of which play crucial roles in the climate system of Mars, using the vantage of the equatorial orbit moderately distant from Mars. Various atmospheric 
phenomena are thought to be involved in water exchange between the atmosphere and the crust, in particular local weather influenced by topography and phase changes in the diurnal cycle. According to numerical simulation of dust entrainment from the surface to the atmosphere, convective plumes with spatial scale $\sim 100 \mathrm{~km}$ and time scales of several hours can play an important role in the onset of dust storms developed to altitudes of $30-50 \mathrm{~km}$ (Spiga et al. 2013). Such localized dust events will induce the transport of water and other trace gases as well. Therefore, it is required to observe the transport of dust, clouds, and water vapor separately with sufficient temporal and spatial resolution (Ogohara et al. 2021). See the "Requirements for spectroscopic imaging" section for details to observe the transported matters separately.

\section{Integrated requirements for observations}

Some of the sensing methods will be applied for multiple scientific purposes, respectively. Thus, detailed requirements for such a sensing method are derived from combinations of related mission requirements. Below, those for geomorphological imaging and laser ranging, spectroscopic imaging, and in situ ion mass spectrometry, which closely relate to multiple mission objectives, will be shown. The requirements for the other observation methods not listed below have been shown in the above subsections: gamma-ray and neutron spectrometry in MR1.1.1 and dust monitoring in MR2.1.

\section{Requirements for geomorphological imaging and laser ranging}

Visible imaging and laser ranging for geomorphology connect to mission requirements under medium objectives $1.1,1.2 \mathrm{a}, 1.3$, and 2.1, respectively. For imaging, it is required to have spatial resolution $<4 \mathrm{~m}(10 \mathrm{~m})$ to identify fresh bedrock exposures at a horizontal scale of $20 \mathrm{~m}$ $(100 \mathrm{~m})$ or better for Phobos (Deimos) as described in MR1.1.1, MR1.3, and MR2.1. To identify the topography of impact craters, the most dominant topographic type on both moons, $\mathrm{S} / \mathrm{N}>30$ is required. This value is sufficient for clear imaging that decomposes the topographic brightness variation on impact craters known to have diverse depth-to-diameter ratios (Hemmi and Miyamoto 2020) when viewing each cratered area from its normal direction under a solar incidence angle of $30^{\circ}$. For sampling operations, it is required to have higher spatial resolutions $<1 \mathrm{~m}$ for each landing-site candidate area $\sim 100 \mathrm{~m}$ across and $<0.1 \mathrm{~m}$ for the area within $\sim 0.5 \mathrm{~m}$ from the sampling point to observe the geologic context of the sampling site.
To make a shape model, imaging at different local times with different viewing angles at each location of Phobos is required. MMX supposes local times for imaging to be around $9.5 \mathrm{~h}, 12.5 \mathrm{~h}$, and $14.5 \mathrm{~h}$ with three different viewing angles at $9.5 \mathrm{~h}$ and $14.5 \mathrm{~h}$ for a better stereo condition (see Matsumoto et al. 2021 for details).

A reliable shape model of Phobos and local 3D elevation maps can be obtained by a synthetic analysis of the imaging and laser ranging data. These are important also for geodetic and geological studies and spectral data mapping. For this purpose, laser ranging is required to be capable of ranging from an altitude of $100 \mathrm{~m}$ to $100 \mathrm{~km}$ which mostly covers the supposed mid-to low altitude range of spacecraft orbit around Phobos (see the "Mission Profile" section). It is also required to have a footprint size $<5 \mathrm{~m}$ with a ranging accuracy $<0.1 \mathrm{~m}$ from $10 \mathrm{~km}$ altitude, a typical altitude of the low orbit, to resolve boulders, small impact craters, and wall structures of grooves which are the subject of geological studies and spectroscopic imaging as shown in the "MR2.1" section.

\section{Requirements for spectroscopic imaging}

Spectroscopic imaging is relevant to mission requirements MR1.1.1, 1.3, 2.1, and 2.3 under medium objectives $1.1,1.3,2.1$, and 2.3 . The wavelength resolution is required to detect absorption features on both moons by hydrous minerals near $0.65 \mu \mathrm{m}$ and $2.7-2.8 \mu \mathrm{m}, \mathrm{H}_{2} \mathrm{O}$ molecules near 3.0-3.2 $\mu \mathrm{m}$, anhydrous minerals near $0.95 \mu \mathrm{m}$, and organic carbon near $3.5 \mu \mathrm{m}$. S/N > 100 at each wavelength region is required to detect subtle differences in absorption features between places on Phobos and Deimos. It is known that the depth of 2.7 and $0.65 \mu \mathrm{m}$ absorption features are smaller than 10\% (Fraeman et al. 2014) and therefore $S / N>100$ is required for precise comparison of the features with an order of $1 \%$ relative absorption enhancement. In addition, the difference in spectrum slope in the visible and near-infrared region between blue and red units of Phobos is also small (mostly less than 10\%; Fraeman et al. 2014) and therefore $S / N>100$ is required to identify the true color of the regions observed. The spatial resolution is required to be $<100 \mathrm{~m}$ for global imaging of both moons and $<20 \mathrm{~m}$ for main areas on Phobos. The best viewing conditions for spectral imaging are phase angle to be around $10^{\circ}$ and local time to be around $12 \mathrm{~h}$. For almost entire areas $\pm 30^{\circ}$ latitudes, observations that meet or approximate the above conditions are required. To observe material distribution nearby each sampling point, it is required to have spatial resolutions $<1 \mathrm{~m}$ for a landing area about $100 \mathrm{~m}$ across and $<5 \mathrm{~mm}$ for the area within $\sim 1 \mathrm{~m}$ from each sampling point while a reduction of wavelength resolution is permissible for the closest imaging. 
Observations of the Martian atmosphere will target atmospheric dust, water vapor, and ice clouds. For mapping dust and ice cloud distribution changes associated with dust storm and topographic wind, $\sim 10 \mathrm{~km}$ spatial resolution and $\sim 1 \%$ precision in scattered solar radiation at visible-infrared bands is required with $\sim 1$-h time resolution during the development of dust storm. For water vapor, to detect and monitor several 100-km-scale heterogeneities (Melchiorri et al. 2007), mapping of column integrated water vapor with an accuracy of $1 \mathrm{pr}-\mu \mathrm{m}$ (precipitable water depth in $\mu \mathrm{m}$ ) and $\sim 100 \mathrm{~km}$ spatial resolution is required. This needs infrared imaging at wavelengths within an $\mathrm{H}_{2} \mathrm{O}$ vapor absorption band and its outsides with $\mathrm{S} / \mathrm{N}>100$ (Ogohara et al. 2021).

\section{Requirements for in situ ion mass spectrometry}

In situ ion mass spectrometry connects to mission requirements in MR1.1.1, MR1.1.3, MR2.1, and MR2.2.2 under medium objectives 1.1, 2.1, and 2.2, with primary observational targets including torus ions derived from $\mathrm{H}_{2} \mathrm{O}$ molecules possibly released from Phobos, secondary ions of major refractory elements $\left(\mathrm{Mg}^{+}, \mathrm{Si}^{+}, \mathrm{Ca}^{+}\right.$, and $\mathrm{Fe}^{+}$) sputtered from Phobos's surface by the solar wind, solar wind ions dominated by $\mathrm{H}^{+}$, and ions escaping from the Martian atmosphere such as $\mathrm{O}^{+}$and $\mathrm{C}^{+}$. Therefore, the ion mass spectrometry is required to have sufficient ion detectability at estimated energy range $(<30 \mathrm{keV})$ and fluxes: water-related ions of $10^{5}-10^{7}$ ions $/ \mathrm{cm}^{2} \mathrm{~s}$ originating from the Phobos torus if exists (Poppe and Curry 2014; Poppe et al. 2016), secondary ions of $\sim 10^{4}$ ions $/ \mathrm{cm}^{2} \mathrm{~s}$ from Phobos (Schaible et al. 2017), solar wind ions of $\sim 10^{8}$ ions $/ \mathrm{cm}^{2} \mathrm{~s}$ (Trotignon et al. 1996) and those scattered at Phobos surface of $10^{5}-10^{6}$ ions $/ \mathrm{cm}^{2}$ s (Futaana et al. 2010), and escaping ions from the Martian atmosphere of $\sim 10^{4}-10^{7}$ ions $/ \mathrm{cm}^{2} \mathrm{~s}$ (Ramstad et al. 2017). It also needs to have sufficient mass resolution to distinguish ion species. To know the direction of ions approaching the spacecraft, which needed to constrain the source of detected ion, simultaneous measurement of the ambient magnetic field in the solar wind with typical strength $3 \mathrm{nT}$ and variable direction is also required. This is because ion motion is restricted by $\boldsymbol{B}$ and $\boldsymbol{E}=-\boldsymbol{V} \times \boldsymbol{B}$ where the vectors $\boldsymbol{B}, \boldsymbol{E}$ and $\boldsymbol{V}$ are the solar wind magnetic field, electric field, and velocity. $1 \mathrm{~Hz}$ sampling is estimated enough to detect magnetic field variations due to change in the solar wind itself and wake behind Mars and Phobos from supposed orbits (Yokota et al. 2021).

\section{Requirements for sampling}

The basic mission requirement for sampling is to collect and bring back samples that can reveal the origin and evolution of Phobos (medium objectives 1.2 and 2.1) and the evolution of the Martian surface environment (2.2).
Specifically, it is necessary to collect samples of $10 \mathrm{~g}$ or more containing material deeper than $2 \mathrm{~cm}$ from two sites while clarifying the geologic context of sampling areas. These are required by MR1.1.2, but also meet the requirements of MR1.2a, 1.2b, MR2.1, and MR2.2.1 for sample analysis. It is also necessary to reduce contamination as much as possible. To prevent contamination of the Earth's atmosphere and Earth's organic matter to volatiles and organic matter in the recovered samples, the contamination control and the sealing of sample container are required following Hayabusa2 (Sawada et al. 2017).

The sampling sites are needed to be sufficiently flat for safe landing and have accessibility to Phobos indigenous materials. Small-scale topographic irregularities on Phobos are studied both by previous images and numerical analyses (Takemura et al. 2021), but analyses at much higher resolutions $\sim 10 \mathrm{~cm}$ are needed. Close-up spectroscopic and geomorphological imaging with spatial resolutions up to the order of $\mathrm{mm}$ is required to describe the geologic context of the sampling site, which allows us to

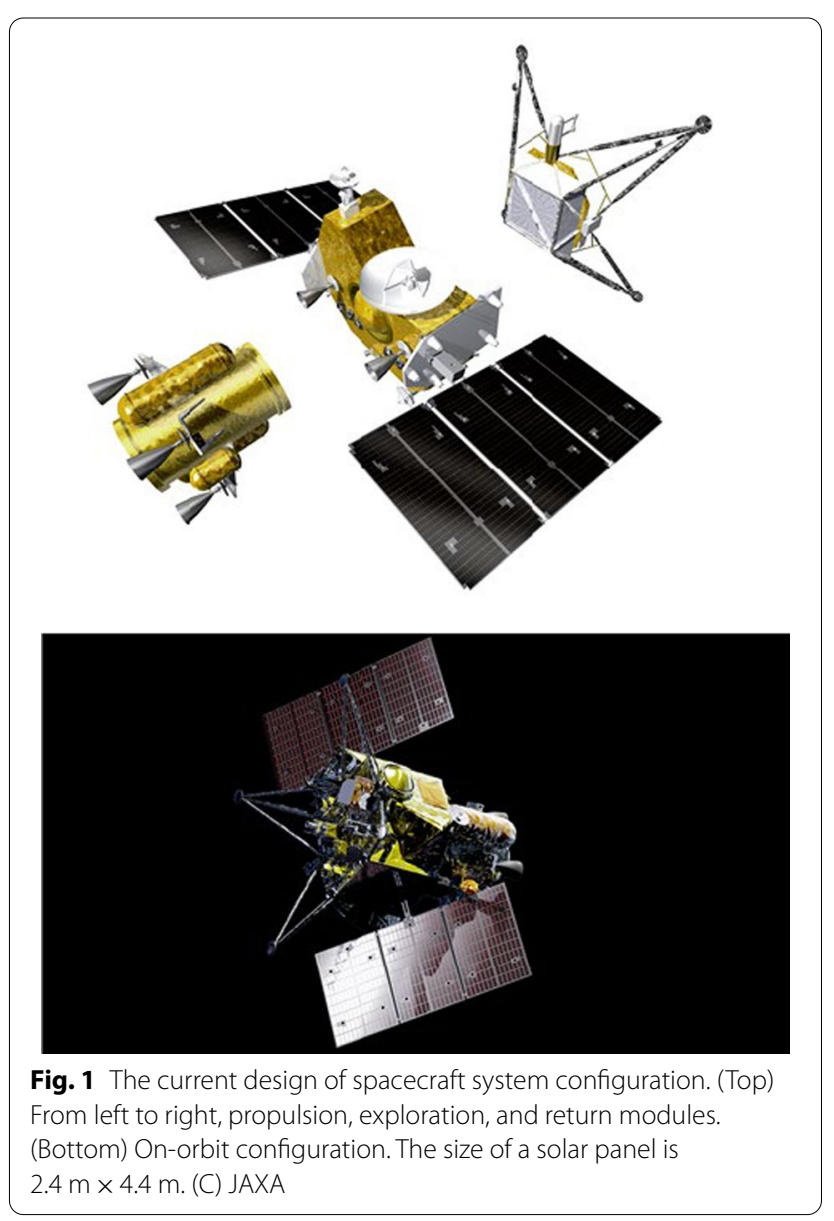


Table 3 Scientific mission objectives and requirements of MMX

\section{Goal 1: Clarify the origins of the Martian moons and constrain processes for planetary formation and material transport in the region} connecting the inner and outer Solar Systems

Medium objective 1.1 Reveal whether Phobos originated as a captured asteroid or resulted from a giant impact

M01.1.1: Spectroscopically reveal the surface-layer distribution of the materials that make up Phobos with the spatial resolution required for the scientific evaluation of sampling points and geological structures, thereby constraining Phobos' origin

M01.1.2: Identify the major components of constituent materials from samples collected on Phobos' surface as Phobos indigenous materials that retain records of their formation, strongly constraining their origins from isotopic ratios, etc.
SS

MR1.1.1: To grasp the distribution of the constituent materials of Phobos, material distributions of hydrous minerals and other related minerals should be obtained spectroscopically for main parts of the full body in correspondence with its topography (at horizontal spatial resolutions of $20 \mathrm{~m}$ or better) and in a radius of $50 \mathrm{~m}$ or more around the sampling point (at spatial resolutions of $1 \mathrm{~m}$ or better). Also, the mean global Si/Fe ratio, etc., of the moon should be determined to within an accuracy of $20 \%$

MR1.1.2: To constrain the origins of Phobos, paying attention to the diversity and representativeness of Phobos' surface, record the occurrence of and collect at least $10 \mathrm{~g}$ of particulate samples (Phobos samples), including samples $2 \mathrm{~cm}$ below the surface. Also, identify main sample components as moon-indigenous materials that were constituent materials at the time of formation, and measures their texture and mineral, elemental, and isotopic composition (oxygen, chromium, etc.) with sufficient accuracy to allow the specification of the moon's origins

M01.1.3: Obtain information such as molecular release rates and mass distribution related to the presence of ice in Phobos, investigate the presence or absence of density contrasts on Phobos'surface, and constrain Phobos' origin independently of MO1.1.1 and MO1.1.2

MR1.1.3: To constrain the origin of Phobos from its internal structure, (1) measure the molecular release rate from internal ice at a detection limit of fewer than $10^{22}$ molecules/s, (2) investigate the presence or absence of inhomogeneity in the density structure due to localization of ice exceeding $10 \%$ of Phobos' mass, and (3) investigate the presence or absence of density variation near the surface layer

Medium objective 1.2a [If Phobos is determined to be a captured asteroid] Elucidate the composition and migration process of primitive materials supplied to the region of terrestrial planets and constrain the initial conditions of Martian surface evolution

M01.2a.1: By constraining the formation of primitive materials $\mathrm{S}$ in the Solar System and primitive bodies in the vicinity of the snow line from a material science perspective, and by estimating the Phobos capture process, constrain the initial conditions for the processes of planetary migration, material transport and evolution of the Martian surface in the early Solar System
MR1.2a: To constrain the initial evolution of Solar System materials and volatile element supply, analyze texture, element and isotope composition, formation age, etc., of moon-indigenous materials in Phobos samples with the necessary accuracy and also extract information related to organic matter and hydrous minerals. Also, elucidate collision environments before and after moon capture from the age distribution of shock alteration in the collected sample and measurements of impact crater distributions on the moon surface

Medium objective 1.2b [lf Phobos is determined to originate from a giant impact] Elucidate giant impact and moon formation processes in the terrestrial planetary region and evaluate its influence on the early evolutionary process of Mars

M01.2b.1: For Phobos indigenous materials, identify primitive $\mathrm{S}$ Martian components (Mars-originating components) ejected by a giant impact and components of the impactor body, clarify their features, estimate the scale and age of the impact, and constrain planetary migration, material transport, and planetary formation processes in the terrestrial planetary region

Medium objective 1.3 Place new constraints on Deimos' origin M01.3.1: Elucidate the surface distribution of materials composing Deimos through spectroscopy with the spatial resolution necessary for grasping its geological structures and compare this with Phobos
MR1.2b: To constrain the process of a giant impact, analyze the texture, elemental and isotopic composition, shock alteration age, etc., of moon-indigenous materials in the Phobos sample with sufficient accuracy, and estimate the peak temperature, timing of the collision, and the mixing ratio of components from primitive Mars and the impacting body. Also, restrict the mixing ratio of both components across the entire moon from measurements of the Si/Fe ratio, etc.

MR1.3: To grasp the distribution of constituent materials of Deimos, from spectroscopic information, clarify the surface distribution of hydrous minerals and other related minerals corresponding to its topography at characteristic parts of the moon with a horizontal spatial resolution of $100 \mathrm{~m}$ or better 
Table 3 (continued)

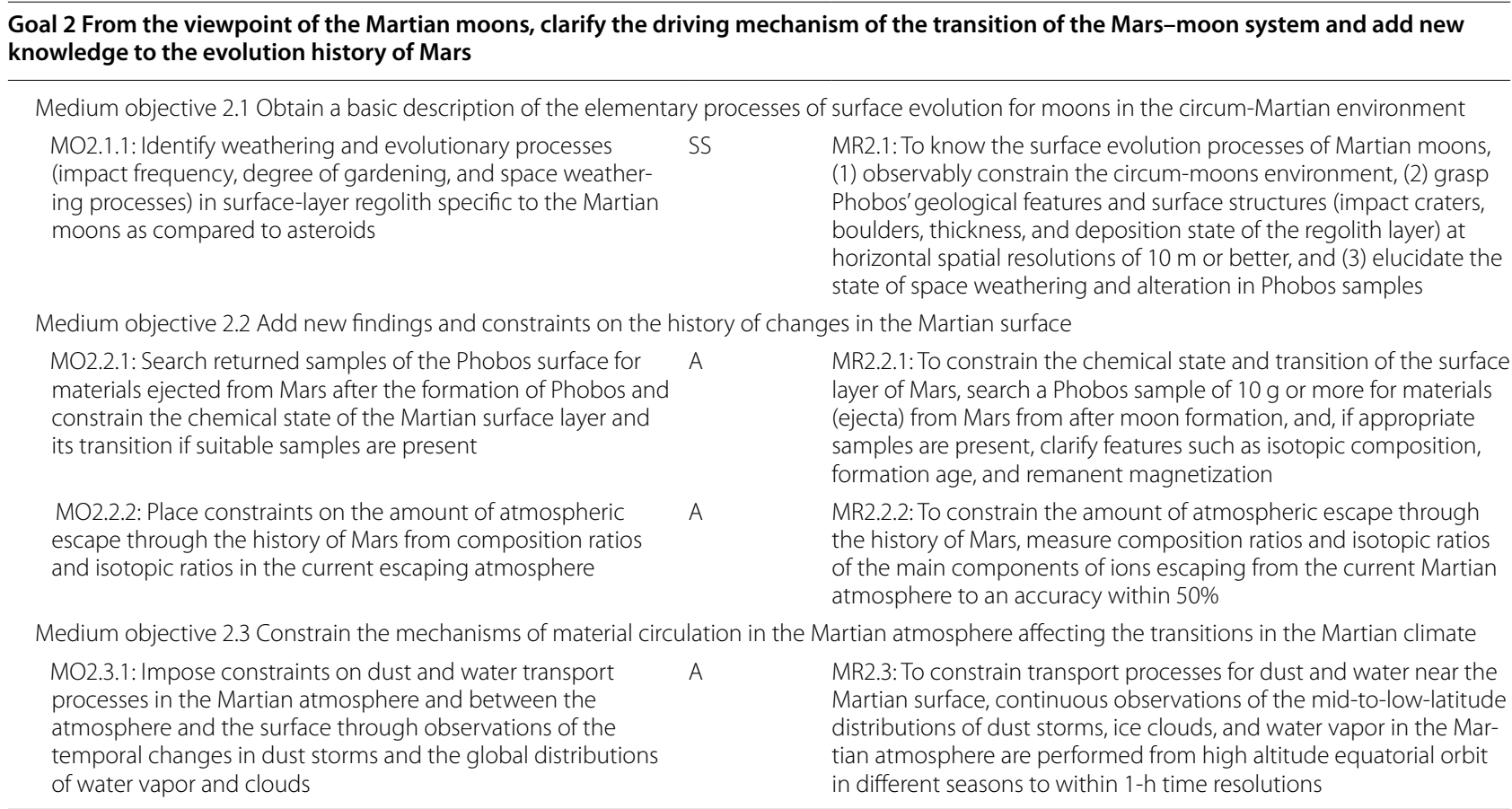

$M O$ mission objectives, $M R$ mission requirements, priority: $S S=$ very high, $S=$ high, $A=$ valuable)

interpret the sample properties connecting with the bulk properties of Phobos.

\section{Spacecraft and science instruments Spacecraft}

Since the design of the MMX spacecraft architecture is reported in detail elsewhere (Kawakatsu et al. 2019), here we briefly summarize its essence. The MMX spacecraft will employ a chemical propulsion system with a high acceleration ability to confine the roundtrip time to a reasonable length (Campagnola et al. 2018). To achieve a round trip to the Martian sphere of gravity, the spacecraft is composed of separable modules: propulsion module, exploration module, and return module (Fig. 1). By releasing used modules at appropriate epochs, the spacecraft mass will be reduced to allow orbital controls in the Martian system and return cruise to the Earth reducing the required overall propellant mass. To carry samples with a volume much larger than that of Hayabusa2 which aimed to take $100 \mathrm{mg}$ of samples (Tsuda et al. 2013), an enlarged return capsule will be used for MMX. The total spacecraft wet mass at launch is about $4000 \mathrm{~kg}$. The spacecraft will be launched by an H-III rocket with launch capabilities larger than $\mathrm{H}$-II rockets. The H-III rocket system is under development as the new Japanese flagship launch vehicle with the planned first launch in 2021.

\section{Science instruments}

Table 4 lists the science instruments aboard MMX. These are selected to satisfy the mission requirements complementary to the sample analyses. A detailed description of each instrument will be given elsewhere. Here, we summarize their complementary roles.

One of the most important roles of proximity observations is to obtain constraints for the origin of the Martian moons independently of sample analyses and also to document the sampling site to inform the geological context. Measurements for the determination of bulk Phobos composition is the most direct approach for this purpose. OROCHI (Kameda et al. 2021), the wide-angle visible multiband camera, and MIRS (Barruci et al. 2021), the infrared spectrometer, will conduct near-global mapping of reflectance spectra of Phobos with spatial resolutions of the order of $10 \mathrm{~m}$. Combining with the specification of fresh bedrock exposures by TENGOO (Kameda et al. 2021), a panchromatic telescopic camera with much higher spatial resolutions, the spectroscopic mapping over wavelength from visible to infrared will constrain the mineral composition of Phobos bedrock. Hydrous minerals, if exist, are a very important proxy indicative of the capture origin of the moon, thus OROCHI and MIRS are designed to detect absorption bands at $\sim 0.7 \mu \mathrm{m}$ and $\sim 2.7 \mu \mathrm{m}$, respectively, caused by hydroxyl group in mineral structures with sufficient signal-to-noise ratio. 
MEGANE (Lawrence et al. 2019), the gamma-ray and neutron spectrometer will determine the concentration of elements such as $\mathrm{Si}, \mathrm{Fe}, \mathrm{H}$, and $\mathrm{K}$ in the surface layer of several tens $\mathrm{cm}$ thickness, where cosmic-ray induced and spontaneous atomic nucleus reactions take place associated with emissions of neutrons and high-energy photons to space, over the hemisphere scale. This instrument is designed to determine $\mathrm{Si} / \mathrm{Fe}$ ratio with the required accuracy (MR1.1.1). It can also determine the $\mathrm{K}$ and $\mathrm{H}$ concentrations if Phobos has a volatile concentration comparable to that of carbonaceous chondrites. MSA (Yokota et al. 2021), the ion mass spectrum analyzer, will attempt to detect the ion torus derived from $\mathrm{H}_{2} \mathrm{O}$ possibly released from Phobos which may store icy materials inside. Metallic ions spattered out from the surface of Phobos by solar wind are also the target of the MSA measurements, which may constrain the elemental composition of Phobos surface averaged over the hemisphere scale as achieved for the Earth`s moon by the Kaguya mission (Yokota et al. 2009).

LIDAR (Senshu et al. 2021), the laser altimeter, will be used for the determination of the shape, rotation, and gravity field of Phobos in combination with the geomorphological imaging by TENGOO and Earth-based spacecraft tracking data. LIDAR will also provide surface roughness information within each laser footprint from analysis of the reflectance as a function of observation angle at the laser wavelength of $1064 \mathrm{~nm}$. This is useful to understand the resurfacing processes such as mass wasting in each geologic unit, concerning the medium mission objective 2.1.

Those remote sensing data will be also used for the selection of sampling sites. The global surface composition of Phobos constrained by proximity observations will be compared with the compositions of the returned sample to examine how returned materials are representative of Phobos indigenous materials. Close-up spectroscopic and panchromatic imaging by OROCHI and TENGOO with spatial resolutions in the order of $\mathrm{mm}$ during landing operations will be used to describe the geologic context of the sampling site.

CMDM (Kobayashi et al. 2018) is a dust detector monitoring impact signals on its sensor of an exposed thin film at the top layer of multi-layer insulation of the spacecraft. It will measure the impact momentum of dust grains colliding with the sensor surface in orbit around Mars. When there is a dust collision on the sensor, it is classified as interplanetary dust, interstellar dust, or Mars orbiting dust based on the information on the orbital position and attitude of the spacecraft at that time. As a result, the flux of those dust species is obtained. Confirmation of the presence or absence of a hypothesized dust rings or tori along the moons' orbits is one of the main purposes of this measurement. The instrument detection limit is set enough to observe micrometeorite flux and expected ring dust as shown in the "MR2.1" section. The measured fluxes of dust grains including meteoroids provide basic data for understanding the weathering and impact gardening processes of both moons. By combining the analyses of samples concerning impact alteration states and ages, mixing of exotic materials, and possible material exchange among bodies in the Martian system, an integrated view for the surface evolution of the Martian moons would be available.

OROCHI and MIRS will also conduct a series of spectroscopic imaging/mapping of the atmosphere of Mars. From positions near Phobos, both instruments are capable of imaging a wide area from mid- to low-latitudes of Mars. At positions more distant from Mars, imaging of the global hemisphere becomes easier. Color imaging by OROCHI can identify ice clouds and atmospheric dust, whereas infrared spectroscopic imaging by MIRS can assess the distribution of the column $\mathrm{H}_{2} \mathrm{O}$ vapor content using the reflectance contrast between the wavelengths in and outside the $\mathrm{H}_{2} \mathrm{O}$ absorption band. By tracing the motion of clouds, atmospheric dust swarms, and $\mathrm{H}_{2} \mathrm{O}$ vapor using the time series of imaging/spectroscopic data, the atmospheric circulation can be monitored. Additionally, zoom-up observations by TENGOO would capture the upwelling of dusty warm air into the upper atmosphere. Ions escaping from the Martian upper atmosphere are detectable by MSA with distinguishing isotopic mass of elements including $\mathrm{O}$ and $\mathrm{C}$.

MMX will carry a rover provided by CNES and DLR for scientific and engineering purposes including being a scout for the lander (Michel et al. 2021). The landing system and landing operation of the mothership have been studied to absorb the uncertainties in the mechanical properties of Phobos surface regolith such as the surface layer strength. On the other hand, it is beneficial to confirm in advance whether the actual regolith properties are within or outside the expected range. The rover will be released before the landing operation and conduct an in situ examination of the Phobos surface. The rover powered by its solar generator will be equipped with navigation cameras, wheels for travel with torque sensors, wheel cameras, imaging the interaction of the wheels with the regolith, a thermal radiation monitor (MiniRad), accelerometers characterizing impact and bouncing, and a laser Raman microscope, RAX (Cho et al. 2021). These instruments will examine mechanical, dynamical, and thermal properties and mineralogy of Phobos surface regolith. The obtained data are also useful as ground truth for interpreting the remote sensing data taken from orbit. 
The sampling system, one of the most important mission instruments of MMX, will conduct sampling from Phobos surface regolith and transfer of samples into a sample container inside the sample return capsule (Kawakatsu et al. 2019). After touch down, sampling will be conducted by using a joint arm manipulator equipped with a coring mechanism at its tip. The MMX mission is planned to make landings at two different sites. At each landing site, a sampling point will be first determined within the area accessible by manipulation based on a precise raw surface image transmitted to the ground station on Earth. An installed corer with an inner diameter of $2 \mathrm{~cm}$ will penetrate to a depth $>2 \mathrm{~cm}$ at the designated point and then the inner sample holder will be transferred to the container space in the return capsule. The corer diameter and penetration depth satisfy the mission requirements on the sampling mass $(\geq 10 \mathrm{~g})$ and depth (MR1.1.2) unless the material porosity is extremely high and/or the density of intact material is much smaller than those of common rocks. During landing operation, OROCHI will conduct spectroscopic imaging of the sampling point and its surroundings with a spatial resolution of the order of mm to specify their spectroscopic property and geologic context. It is technically difficult to take spectroscopic images resolving individual grains with the estimated size range $(300 \mu \mathrm{m}-2 \mathrm{~mm})$. However, the penetration area of the sampler core can be resolved, then the average spectral characteristics of the sampling point can be ascertained in comparison with surrounding materials.

A pneumatic sampler, another type of sampler provided by NASA applying the same working principle as used during the OSIRIS-Rex mission (Bierhaus et al. 2018), installed at a landing pad will also attempt sampling of regolith at shallow depth (Kawakatsu et al. 2019). Samples taken from different depths are useful to understand the possible layering of surface regolith caused by space weathering, the influx of exotic materials, and gardening by meteoroid impacts (Usui et al. 2020). We call this sampler P-sampler, whereas the primary coring sampler is called $\mathrm{C}$-sampler. The sample holder of the $\mathrm{P}$-sampler will be transported to the container space in the return capsule by the manipulator.

\section{Mission profile \\ Overall}

The planned mission profile is shown in Fig. 2. The MMX spacecraft is planned to be launched in September 2024 and reach the Martian system after the cruise slightly less than a year. The spacecraft will stay in the Martian system for nearly 3 years and conduct close-up observations of both moons, delivery of the rover, and sampling from Phobos. The spacecraft will depart the Martian system in
August 2028 and return the capsule containing collected samples to the Earth.

After the insertion to a circum-Martian orbit, the spacecraft motion will be tuned to approximate the Phobos motion. By taking small differences in orbital eccentricity from Phobos, the spacecraft will eventually circulate Phobos at small distances. In the frame fixed to Phobos, the spacecraft trajectory will form an ellipse, or quasi-satellite orbit (QSO), with the center at the Phobos mass center (Fig. 3). The semimajor axis of QSO, which is perpendicular to the Mars direction, is about twice the minor axis. Decreasing in separation from Phobos, QSO gradually approaches to circle, or the semimajor axis/ minor axis ratio decreases, due to the greater contribution of Phobos gravity.

MMX will utilize several QSO patterns with high (semimajor axis $200 \mathrm{~km}$, QSO-H), medium (100 km, QSO$\mathrm{M}$ ), and low (50 km and below, QSO-L series) altitudes depending on the observation phase. The orbital periods of QSOs are shorter than the revolution period of $7.65 \mathrm{~h}$ due to the effect of this moon's gravity (7.1 h for QSO-M, $\leq 5.7 \mathrm{~h}$ for QSO-L series; Canalias et al. 2017). When the spacecraft is staying in a QSO, the local time at the spacecraft passing above a fixed longitude on Phobos progressively drifts with rates depending on QSO radius, which allows observations of the same area under different solar illumination angles.

MMX will also introduce the so-called 3D-QSO with inclination to Phobos, which allows observation of highlatitude regions of this moon (Fig. 3b). The parameter setting of 3D-QSO is yet to be fixed while the orbital radius comparable to QSO-M with the maximum separation from the equatorial plane $\geq 30 \mathrm{~km}$ which allows imaging of polar regions is supposed as a baseline. From QSOs, MMX will carry out observations of Phobos including those for landing site selection, circum-Martian space, and the Martian atmosphere.

During the period in proximity to Phobos, the spacecraft will land on two different sites of Phobos. Since Phobos's surface gravity is two orders of magnitude larger than those of Itokawa and Ryugu, the descent and landing sequence of MMX spacecraft is different from the prior missions Hayabusa and Hayabusa2 which used a so-called touch and go approach for sampling operations. For example, a low-velocity descent from a very high altitude cannot be adopted considering fuel consumption to keep low velocity against gravity. Instead, from low QSO orbit, a ballistic descent will be applied to reach $\sim 2 \mathrm{~km}$ above a landing site before starting the subsequent final vertical descent. A rehearsal operation before landing will take close-up images of the area including landingsite candidates to confirm the safety and the availability of samples that are representative of bedrock materials. 


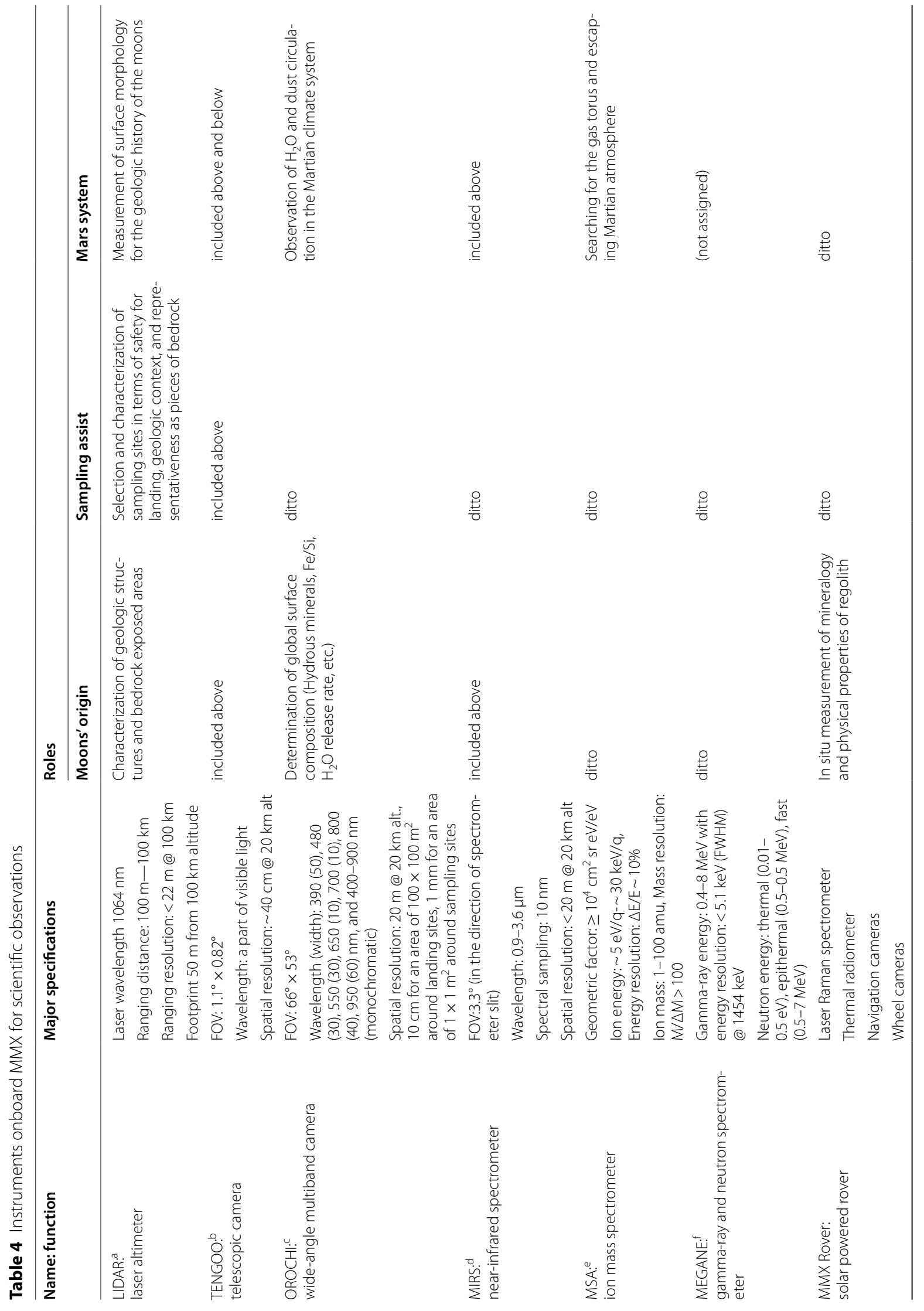




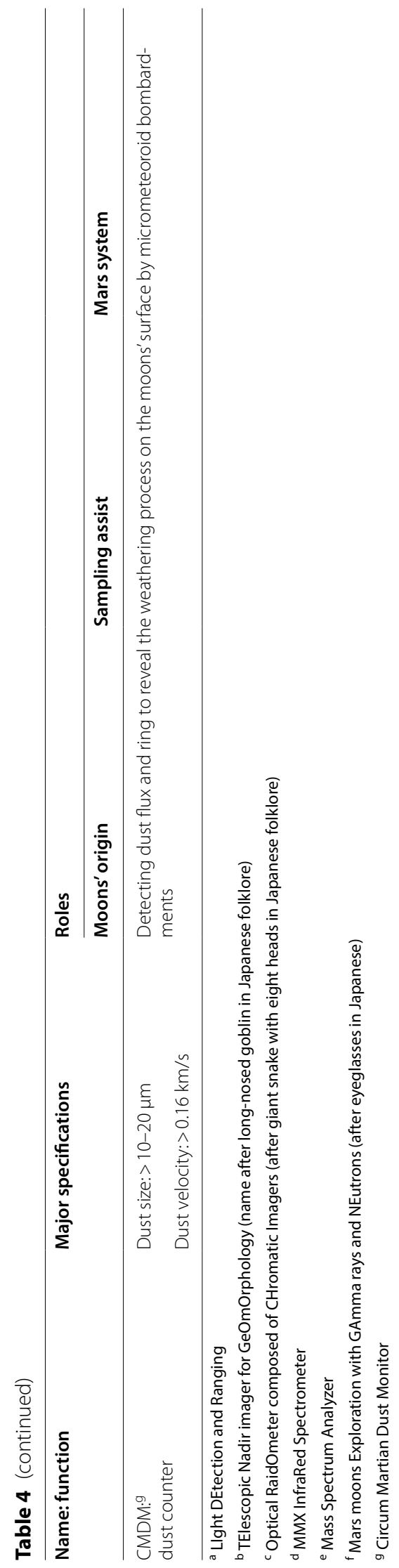




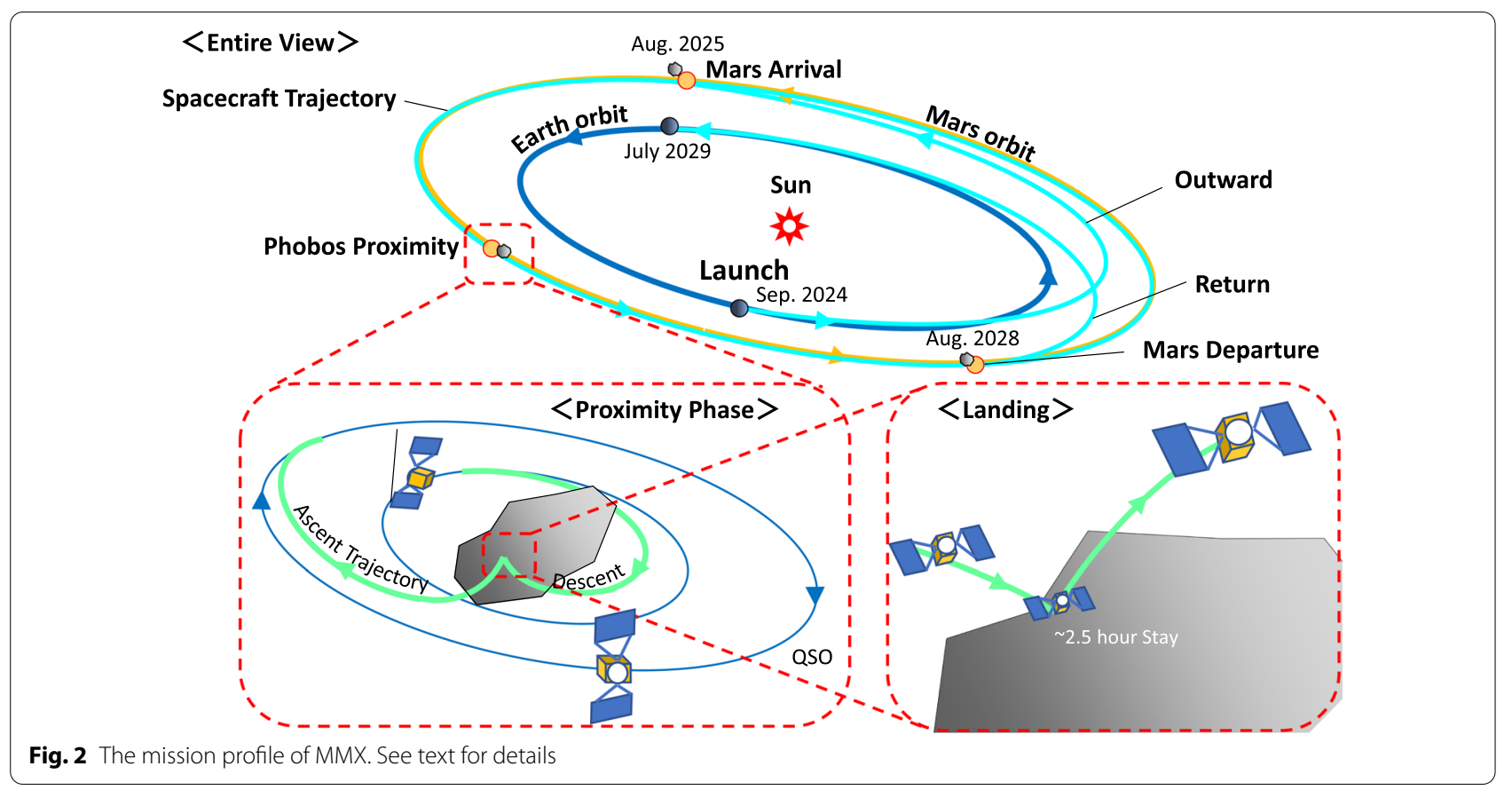

To avoid previously unrecognized obstacles such as large boulders in the area of the selected site, an autonomous hazard detection, and avoidance technology will also be set up to realize a safe landing. The landing gear is installed with a mechanism absorbing the spacecraft momentum relative to the surface to prevent the spacecraft from bouncing at the touch down (Kawakatsu et al. 2019).

After the completion of proximity observations of Phobos and sampling operations, the spacecraft will expand its orbit to perform multiple flybys of Deimos. During this period, close-up observations of Deimos will be conducted together with those of the surrounding space and the Martian atmosphere. Then, the spacecraft will depart from the Martian system.

\section{Operations during the stay around the Martian moons}

In the nominal operation plan during the stay in the Martian system (Fig. 4), solar conjunctions will occur in January 2026 and March 2028. Science operations may be inhibited for $\sim 1.5$ months around these conjunction timings. Furthermore, Mars will arrive at an equinox in late November 2025 and this will happen subsequently after every about half of the Martian year. During the periods of about 3 months with equinox timing in each center, a spacecraft on QSO-L around Phobos will experience eclipses with long duration by Mars and Phobos repeatedly. This is caused by the near-equatorial orbit of Phobos around Mars and relatively small differences in QSO periods from the length of the day of Phobos. For maintaining the health of electric devices, long stops of power generation should be avoided. During those periods around equinoxes, therefore, QSO operation is restricted to large elliptic orbit with minor and major radii no smaller than $50 \mathrm{~km}$ and $100 \mathrm{~km}$ around Phobos to reduce the duration of every eclipse.

The current plan for observational operation is shown in detail elsewhere (Nakamura et al. 2021), here we briefly overview it below. Considering the constraints shown above, the period of $\sim 3$ years stay in the sphere of Martian gravity is divided into five phases as shown in Fig. 4. Phase 1 starts from MOI (Mars Orbit Insertion). After MOI, the spacecraft will be transferred to QSO around Phobos after the deployment of the propulsion module. During this transfer, the spacecraft will have a chance to approach Deimos at outer orbit, which would be used as an opportunity for the first Deimos imaging observation from small distances. After insertion to QSO, observations of Phobos from QSO-H will be conducted for the global shape model generation and spectral mapping of Phobos.

During phase 2 that starts after the first solar conjunction, close-up observations from QSO-M and QSO-L series will be combined to conduct higher resolution imaging and spectral mapping. Observations from QSO-LC are essential for the gamma-ray and neutron spectrometry by MEGANE. Preliminary study on the data acquisition and transmission rates using simulated time series of spacecraft orbits and orientations according to the tentative operation sequence in Fig. 4 shows 


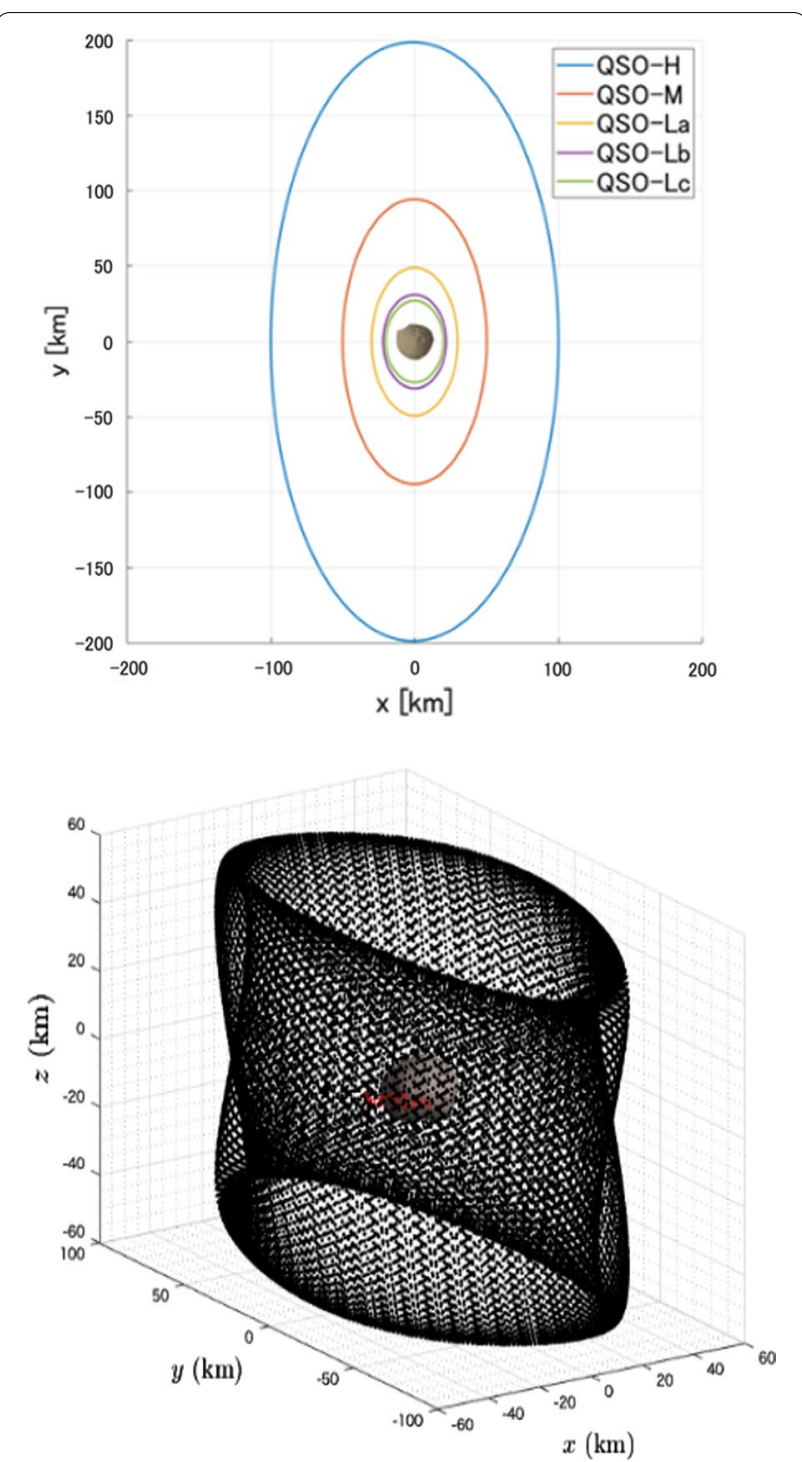

Fig. 3 Planned observational orbits around Phobos. Quasi-satellites orbits (QSOs) are drawn in the Phobos fixed frame with Phobos at the center taking the $x y$-plane and the $x$-axis direction to be the Phobos orbital plane and the opposite direction to Mars, respectively. Top: QSOs confined in the xy-plane. Bottom: An example of 3D-QSO trajectory for the case taking effective orbital radius same as QSO-M and inclination of $45^{\circ}$

that global geomorphological-topographic mapping and spectrometries of Phobos satisfying the corresponding mission requirements can be almost completed during phases 1 and 2 (Nakamura et al. 2021).

The descent and landing operations will be performed during phase 3 using the advantage in solar illumination conditions and short Earth-Mars distance. The release of the rover and the descent and ascent operation, which is a part of the landing rehearsal, will be done before the first actual landing but their best timings are under study including the last part of phase 2 . Observations with progressive improvement of spatial resolution during phases 1 and 2 will be used for the landing site selection to guarantee the safety for landing and the accessibility to indigenous materials. The landings (represented by TD1 and TD2 in Fig. 4) are supposed to be done at near- and antiMars sides, respectively. Its details including the order of near- and far-side landings are under study.

A landing operation that includes touch down, sampling, and departure will be completed during a single daytime of Phobos lasting $3 \mathrm{~h} 50 \mathrm{~min}$. The stay time on the surface is planned to be $2.5 \mathrm{~h}$ with about a $1-\mathrm{h}$ margin in the entire daytime. The time duration allocated for the sampling operation is $1.5 \mathrm{~h}$, which includes close-up imaging of the landing area to determine the sampling point by the $\mathrm{C}$-sampler and the telemetries of image data and command with the control station on the Earth. The P-sampler system will be used only during the first landing.

\section{Sample analyses}

Since the current strategy of MMX sample analyses is reported in detail elsewhere (Usui et al. 2020; Fujiya et al. 2021), here we briefly summarize its essence.

Returned samples, expected to be granular as previously mentioned, will be first subjected to screening under an optical microscope analysis which observes grain size, shape, texture, and crude mineralogy. Selected grains will be analyzed using Raman spectroscopy and Fourier-transform infrared spectroscopy (FT-IR) to characterize precise mineralogy and chemical bonds of constituents including organics. For grains containing magnetic minerals, magnetometry using a superconducting quantum interference device (SQUID) will be also applied. Further mineralogical observations and qualitative analyses of chemical compositions will be carried out using scanning electron microscopy (SEM) with energydispersive X-ray spectrometry (EDS).

The estimated typical grain size from $300 \mu \mathrm{m}$ to $2 \mathrm{~mm}$ is large enough for the application of a series of precise analysis techniques including X-ray diffraction (XRD) analyses, X-ray microtomography, successive 3D-computed tomography (3D-CT), X-ray absorption near-edge structure (XANES) analyses to assess mineralogy, grain texture, and porosity. After the application of a series of non-destructive analyses, grains larger than $1 \mathrm{~mm}$ will also be used for analyses including secondary ion mass spectrometry (SIMS), and laser-ablation (LA) inductively coupled-plasma mass spectrometry (ICP-MS) to reveal isotopic characteristics and age information. For organics-bearing grains, which is highly expected for the case of capture origin of Phobos, a series of volatile analyses 


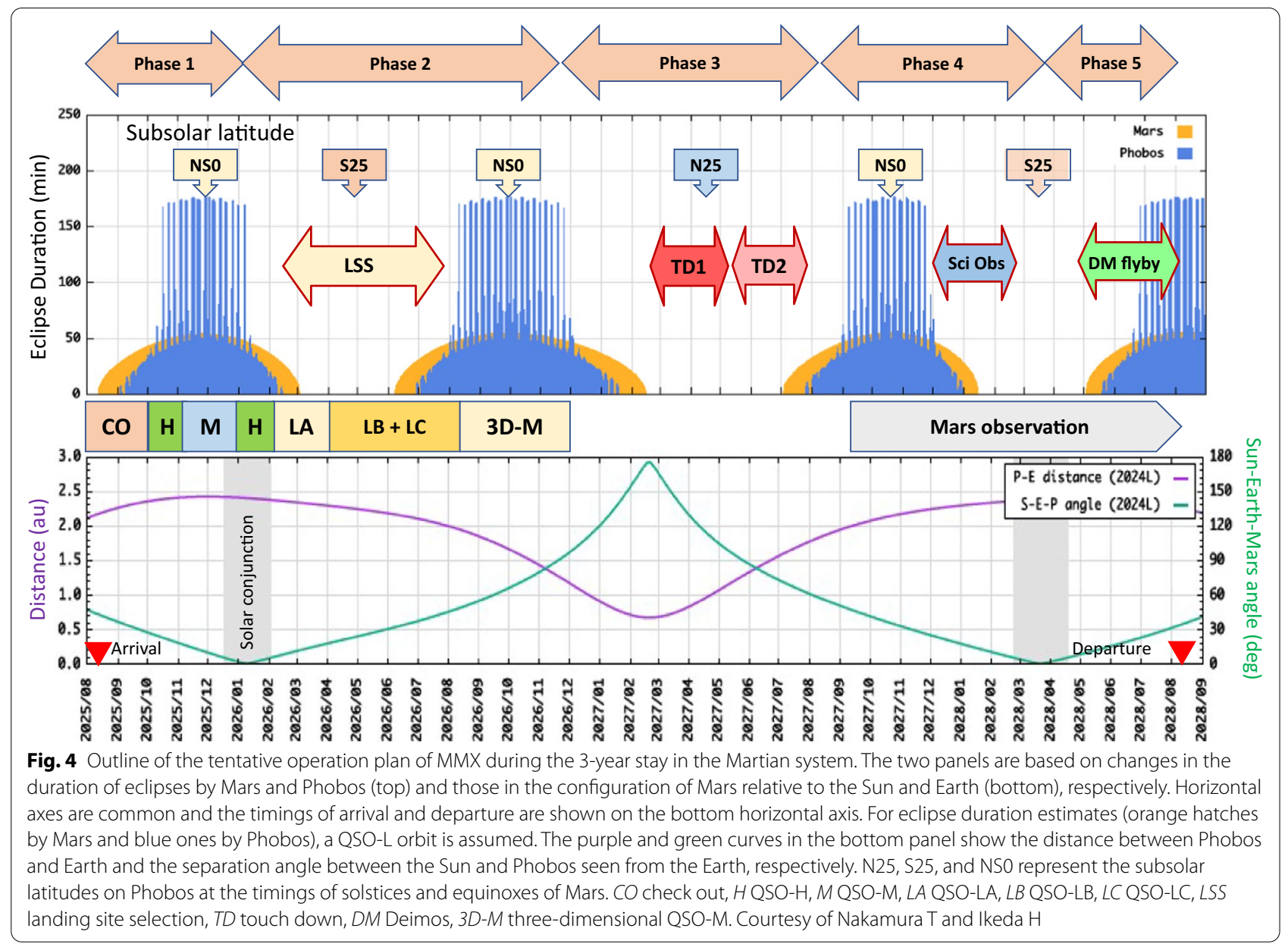

including cavity ring-down laser absorption spectroscopy (CRDS) and gas chromatography/mass spectrometry will be applied depending on the gain mass and volatile concentration. See Fujiya et al. (2021) for details including other analysis techniques to be applied.

By 2029, when Phobos samples are scheduled to be returned, sample analysis techniques will have more advanced. Recently, the Hayabusa2 spacecraft returned Ryugu samples of more than $5 \mathrm{~g}$, far exceeding the original target of $0.1 \mathrm{~g}$. Currently, the first experience in handling return samples from a small body containing water and organics is being gained by the curation team and collaboration (JAXA Hayabusa2 Project 2021). Along with the forthcoming analyses of Bennu samples to be returned in 2023, this will provide new insights into the formation and evolution of primitive materials, as well as the advancement of know-how and techniques for sample curation and multifaceted analyses, which will be applied to Phobos samples. The analyses of Phobos samples will complement the findings and constraints from the smallbody explorations and enhance our understanding of material transport and evolution to create habitable planets in the early Solar System.

\section{Concluding remarks}

Through the exploration of the Marian moons, the MMX mission extends the experience in Japanese sample return exploration for the small bodies and will investigate key processes for the formation and evolution of habitable planets having atmosphere and water. Close-up observations of both moons and detailed analyses of samples returned from Phobos will characterize the properties of their constituent materials in great detail. The mission will determine the origin of the moons which is currently under debate, with models of the capture of primordial, carbonaceous asteroids and the accumulation of circumMartian debris ejected by a huge impact on early Mars both being seriously considered. Also, processes for the acquisition of water, organics, and volatiles from the outer Solar System by early terrestrial planets will be 
elucidated. If the giant impact origin is the case, the differentiation state of a proto-planet mantle just before the occurrence of the moon-forming impact will also be a study target.

Sample analyses will also extract records on the longterm evolutionary processes such as continuous impactor flux and irradiation of solar wind, cosmic rays, and circum-Martian plasma. For this issue, it is beneficial to conduct multidisciplinary studies with remote and in situ data including the impact crater population and surface reflectance of the moons and the dust and plasma environment in circum-Martian space. Concurrent evolutionary processes such as atmospheric circulation and escape and the possible formation of dust ring and gas torus along the moons' orbits will also be study targets. Ejecta materials derived from young Martian impact craters, if found in the returned samples, will provide unique information on the Martian surface environment at the time of impact and/or bedrock formation before impact.

The status of the MMX mission is now in phase $\mathrm{C}$ as a project of JAXA. Targeting the launch in 2024, the detailed design of spacecraft systems and instruments as well as studies of spacecraft orbits, operations, and data processing including basic experiments are extensively ongoing.

\section{Acknowledgements \\ We thank the MMX study team, the MMX project team, the sub-science teams (Origin of Phobos and Deimos, Early Solar System Evolution, Surface Science and Geology, Mars Science, and Geodesy), the instrument teams (CMDM, C-Sampler, LIDAR, MEGANE, MIRS, MSA, ROVER, and TENGOO-OROCHI), and the working teams (Data Processing, Landing Operation, Landing Site Selec- tion, Mission Operation, Mission Operation Preparation, Rover Operation, and Sample Analysis) for insightful discussions for this project. We greatly appreciate N. Namiki and an anonymous reviewer for their careful reading and constructive comments.}

\section{Authors' contributions}

Overall conceptualization: KK, YK, MF, TU, and KW. Science backgrounds: GH, TI, KM, HM, TM, TN, KO, NT, and SW. Origin of Phobos and Deimos: TN and HG. Surface science and geology: HM and KW. Mars science: TI, NT, and HN Geodesy: KM and HS. Spacecraft and instrument system: $Y K, H O, K O$, and $M O$. LIDAR: HS (CIT) and KM. TENGOO/OROCHI: SK and MO. MIRS:MAB, HN, and TN, MEGANE: DJL, TU, KO and HK, MSA:SY and NT, CMDM: MK and SS. Rover: PM and SU. Sampler: HS (JAXA) and TU. Scientific operation and orbital design: TN, HS (CIT), and HI. Sample sciences: TU, ST, and SR. All authors discussed the study and commented on the manuscript. All authors read and approved the final manuscript.
\end{abstract}

\section{Funding}

Through the Phase-A and Phase-B activities of MMX, our work was supported by ISAS/JAXA, NASA, CNES, DLR, and ESA. Parts of studies are also supported by JSPS KAKENHI Grant Number 18 K03719 and 21 K03638 to KK and MEXT KAKENHI Grant Number $17 \mathrm{H} 06457$ and 17H06459 to HG and TU, respectively.

Availability of data and materials

Not applicable.

\section{Declarations}

\section{Competing interests}

The authors declare that they have no competing interests.

\section{Author details}

${ }^{1}$ Hokkaido University, Sapporo, Japan. ${ }^{2}$ Institute of Space and Astronautical Science, Japan Aerospace Exploration Agency, Sagamihara, Japan.

${ }^{3}$ The University of Tokyo, Tokyo, Japan. ${ }^{4}$ Laboratoire d'Etudes Spatiales Et d'Instrumentation en Astrophysique, Paris Observatory, Meudon, France. ${ }^{5}$ Tokyo Institute of Technology, Tokyo, Japan. ${ }^{6}$ Aizu University, Aizu Wakamatsu, Japan. ${ }^{7}$ Research and Development Directorate, Japan Aerospace Exploration Agency, Sagamihara, Japan. ${ }^{8}$ Deutsches Zentrum für Luft- und Raumfahrt, Berlin, Germany. ${ }^{9}$ Rikkyo University, Tokyo, Japan. ${ }^{10}$ Chiba Institute of Technology, Narashino, Japan. ${ }^{11}$ National Institutes for Quantum and Radiological Science and Technology, Chiba, Japan. ${ }^{12}$ Johns Hopkins University Applied Physics Laboratory, Laurel, USA. ${ }^{13}$ National Astronomical Observatory of Japan, Oshu, Japan. ${ }^{14}$ The Graduate University for Advanced Studies, SOKENDAl, Hayama, Japan. ${ }^{15}$ Université Côte d'azur, Observatoire de La Côte d'Azur, CNRS, Laboratoire Lagrange, Nice, France. ${ }^{16}$ Tohoku University, Sendai, Japan. ${ }^{17}$ JAXA Space Exploration Center, Japan Aerospace Exploration Agency, Sagamihara, Japan. ${ }^{18}$ Natural History Museum, London, UK. ${ }^{19}$ Osaka University, Toyonaka, Japan. ${ }^{20}$ Deutsches Zentrum für Luft- und Raumfahrt, Cologne, Germany. ${ }^{21}$ Nagoya University, Nagoya, Japan.

Received: 26 January 2021 Accepted: 11 November 2021

Published online: 20 January 2022

\section{References}

Altwegg K, Balsiger H, Bar-Nun A, Berthelier JJ, Bieler A, Bochsler P, Briois C, Calmonte U, Combi M, De Keyser J, Eberhardt P, Fiethe B, Fuselier S, Gasc S, Gombosi TI, Hansen KC, Hässig M, Jäckel A, Kopp E, Korth A, LeRoy L, Mall U, Marty B, Mousis O, Neefs E, Owen T, Rème H, Rubin M, Sémon T, Tzou C-Y, Waite H, Wurz P (2015) 67P/ChuryumovGerasimenko, a Jupiter family comet with a high D/H ratio. Science 347:1261952. https://doi.org/10.1126/science.1261952

Amsellem E, Moynier F, Mahan B, Beck P (2020) Timing of thermal metamorphism in CM chondrites: Implications for Ryugu and Bennu future sample return. Icarus 339:113593. https://doi.org/10.1016/j.icarus. 2019.113593

Asphaug E, Melosh HJ (1993) The stickney impact of Phobos: a dynamical model. Icarus 101:144-164. https://doi.org/10.1006/icar.1993.1012

Barruci MA, Reess JM, Bernardi P, Doressoundiram A, Fornaiser S, Du ML, Iwata T, Nakagawa H, Nakamura T, Andre Y, Aoki S, Arai T, Baldit E, Beck P, Buey JT, Canalias E, Castelnau M, Charnoz S, Chaussidon M, Chapron F, Ciarletti V, Delbo M, Dubois B, Gauffre S, Gautier T, Genda H, Hassen-Khodja R, Hervet G, Hyodo R, Imbert C, Imamura T, Jorda L, Kameda S, Kouach D, Kouyama T, Kuroda T, Kurokawa H, Lapaw L, Lasue J, Deit LL, Ledot A, Leyrat C, Ruyet BL, Matsuoka M, Merlin F, Miyamoto H, Moynier F, Tuong NN, Ogohara K, Osawa T, Parisot J, Pistre L, Quertier B, Raymond S, Rocard F, Sakanoi T, Sato TM, Sawyer E, Tache F, Tremolieres S, Tsuchiya F, Vernazza P, Zeganadin D (2021) MIRS an imaging spectrometer for the MMX mission. Earth Planets Space. https://doi.org/10.1186/s40623-021-01423-2

Basilevsky AT, Lorenz CA, Shingareva TV, Head JW, Ramsley KR, Zubarev AE (2014) The surface geology and geomorphology of Phobos. Planet Space Sci 102:95-118. https://doi.org/10.1016/j.pss.2014.04.013

Bibring JP, Langevin Y, Mustard JF, Poulet F, Arvidson R, Gendrin A, Gondet B, Mangold N, Pinet P, Forget F, Berthe M, Bibring JP, Gendrin A, Gomez C, Gondet B, Jouglet D, Poulet F, Soufflot A, Vincendon M, Combes M, Drossart P, Encrenaz T, Fouchet T, Merchiorri R, Belluci G, Altieri F, Formisano V, Capaccioni F, Cerroni P, Coradini A, Fonti S, Korablev O, Kottsov V, Ignatiev N, Moroz V, Titov D, Zasova L, Loiseau D, Mangold N, Pinet P, Doute S, Schmitt B, Sotin C, Hauber E, Hoffmann H, Jaumann R, Keller U, Arvidson R, Mustard JF, Duxbury T, Forget F, Neukum G (2006) Global mineralogical and aqueous Mars history derived from OMEGA/Mars Express data. Science 312:400-404. https://doi. org/10.1126/science.1122659 
Bierhaus EB, Clark BC, Harris JW, Payne KS, Dubisher RD, Wurts DW, Hund RA, Kuhns RM, Linn TM, Wood JL, May AJ, Dworkin JP, Beshore E, Lauretta DS, OSIRIS-REX Team (2018) The OSIRIS-REx spacecraft and the touchand-go sample acquisition mechanism (TAGSAM). Space Sci Rev 214:107. https://doi.org/10.1007/s11214-018-0521-6

Brain D, Barabash S, Boesswetter A, Bougher S, Brecht S, Chanteur G, Hurley D, Dubininh E, Fang X, Fraenz M, Halekas J, Harnett E, Holmstrom M, Kallio E, Lammer H, Ledvina S, Liemohn M, Liu K, Luhmann J, Ma Y, Modolon R, Nagy A, Motschmann U, Nilsson H, Shinagawa H, Simon S, Terada N (2010) A comparison of global models for the solar wind interaction with Mars. Icarus 206:139-151. https://doi.org/10.1016/j. icarus.2009.06.030

Burns JA (1972) Dynamical characteristics of Phobos and Deimos. Rev Geophys 10:463-483. https://doi.org/10.1029/RG010i002p00463

Burns JA (1978) The dynamical evolution and origin of the Martian moons. Vistas Astron 22:193-210. https://doi.org/10.1016/0083-6656(78) 90015-6

Campagnola S, Yam CH, Tsuda Y, Ogawa N, Kawakatsu Y (2018) Mission analysis for the Martian Moons Explorer (MMX) mission. Acta Astronaut 146:409-417. https://doi.org/10.1016/j.actaastro.2018.03.024

Canalias E, Lorda L, Martin T, Laurent-Varin J, Marty JC, Mimasu Y (2017) Trajectory analysis for the Phobos proximity phase of the MMX mission. In: international symposium on space technology and science, ISTS-2017-d-006, Ehime, Japan, pp. 3-9.

Canup R, Salmon J (2018) Origin of Phobos and Deimos by the impact of a Vesta-to-Ceres sized body with Mars. Sci Adv 4:aar6887. https://doi. org/10.1126/sciadv.aar6887

Carr MH (1996) Water on Mars. Oxford University Press, New York

Chassefière E, Leblanc F (2004) Mars atmospheric escape and evolution; interaction with the solar wind. Planet Space Sci 52:1039-1058. https://doi.org/10.1016/j.pss.2004.07.002

Cho Y, Bottger U, Rull F, Belenguer T, Borner A, Buder M, Bunduki Y, Dietz E, Hagelschuer T, Hubers H-W, Kameda S, Kopp E, Lieder M, Lopez G, Moral Inza A, Paproth C, Perez Canora C, Pertenais M, Peter G, Prieto Ballesteros O, Rockstein S, Rodd-Routley S, Rodriguez Perez P, Ryan C, Santamaria P, Sauberlich T, Schrandt F, Schroder S, Stangarone C, Ulamec S, Usui T, Weber I, Westerdorff K, Kuramoto K (2021) In-situ science on Phobos with the Raman spectrometer for MMX (RAX): preliminary design and feasibility of Raman measurements. Earth Planets Space. https://doi.org/10.1186/s40623-021-01496-z

Christou AA, Oberst J, Lupovka V, Dmitriev V, Gritsevich M (2014) The meteoroid environment and impacts on Phobos. Planet Space Sci 102:164-170. https://doi.org/10.1016/j.pss.2013.07.012

Citron Rl, Genda H, Ida S (2015) Formation of Phobos and Deimos via a giant impact. Icarus 252:334-338. https://doi.org/10.1016/j.icarus. 2015.02.011

Clayton RN (1993) Oxygen isotopes in meteorites. Annu Rev Earth Planet Sci 21:115-149. https://doi.org/10.1146/annurev.ea.21.050193.000555

Clement MS, Kaib NA, Raymond SN, Walsh KJ (2018) Mars' growth stunted by an early giant planet instability. Icarus 311:340-356. https://doi. org/10.1016/j.icarus.2018.04.008

Craddock RA (2011) Are Phobos and Deimos the result of a giant impact? Icarus 211:1150-1161. https://doi.org/10.1016/j.icarus.2010.10.023

Dauphas N, Pourmand A (2011) Hf-W-Th evidence for rapid growth of Mars and its status as a planetary embryo. Nature 473:489-492. https:// doi.org/10.1038/nature10077

Debaille V, Brandon AD, Yin QZ, Jacobsen B (2007) Coupled ${ }^{142} \mathrm{Nd}{ }^{143} \mathrm{Nd}$ evidence for a protracted magma ocean in Mars. Nature 450:525-528. https://doi.org/10.1038/nature06317

DeMeo FE, Carry B (2014) Solar System evolution from compositional mapping of the asteroid belt. Nature 505:629-634. https://doi.org/10. 1038/nature12908

Duxbury TC, Zakharov AV, Hoffmann H, Guinness EA (2014) Spacecraft exploration of Phobos and Deimos. Planet Space Sci 102:9-17. https://doi.org/10.1016/j.pss.2013.12.008

Fanale FP, Salvail JR (1990) Evolution of the water regime of Phobos. Icarus 88:380-395. https://doi.org/10.1016/0019-1035(90)90089-R
Fernandes VA, Fritz J, Weiss BP, Garrick-Bethell I, Shuster DL (2013) The bombardment history of the Moon as recorded by ${ }^{40} \mathrm{Ar}-{ }^{39} \mathrm{Ar}$ chronology. Meteorit Planet Sci 48:241-269. https://doi.org/10.1111/maps.12054

Fraeman AA, Murchie SL, Arvidson RE, Clark RN, Morris RV, Rivkin AS, Vilas F (2014) Spectral absorptions on Phobos and Deimos in the visible/ near infrared wavelengths and their compositional constraints. Icarus 229:196-205. https://doi.org/10.1016/j.icarus.2013.11.021

Fraeman AA, Arvidson RE, Murchie SL, Rivkin A, Bibring JP, Choo TH, Gondet B, Humm D, Kuzmin RO, Manaud N, Zabalueva EV (2012) Analysis of disk-resolved OMEGA and CRISM spectral observations of Phobos and Deimos. J Geophys Res 117:E00J15. https://doi.org/10.1029/ 2012JE004137

Fujiwara A, Kawaguchi J, Yeomans DK, Abe M, Mukai T, Okada T, Saito J, Yano H, Yoshikawa M, Scheeres DJ, Barnouin-Jha O, Cheng AF, Demura H, Gaskell RW, Hirata N, Ikeda H, Kominato T, Miyamoto H, Nakamura AM, Nakamura R, Sasaki S, Uesugi K (2006) The rubble-pile asteroid Itokawa as observed by Hayabusa. Science 312:1330-1334. https:// doi.org/10.1126/science.1125841

Fujiya W, Sugiura N, Hotta H, Ichimura K, Sano Y (2012) Evidence for the late formation of hydrous asteroids from young meteoritic carbonates. Nat Commun 3:627. https://doi.org/10.1038/ncomms1635

Fujiya W, Furukawa Y, Sugahara H, Koike M, Bajo K, Chabot NL, Miura YN, Moynier F, Russell SS, Tachibana S, Takano Y, Usui T, Zolensky ME (2021) Analytical protocols for Phobos regolith samples returned by the Martian Moons eXploration (MMX) mission. Earth Planet Space 73:120. https://doi.org/10.1186/s40623-021-01438-9

Futaana Y, Barabash S, Holmström M, Fedorov A, Nilsson H, Lundin R, Dubinin E, Fränz M (2010) Backscattered solar wind protons by Phobos. J Geophys Res 115:A10213. https://doi.org/10.1029/2010J A015486

Genda H (2016) Origin of Earth's oceans: an assessment of the total amount, history and supply of water. Geochem J 50:27-42. https://doi.org/10. 2343/geochemj.2.0398

Gendrin A, Langevin Y, Erard S (2005) ISM observation of Phobos reinvestigated: identification of a mixture of olivine and low-calcium pyroxene. J Geophys Res Planets 110:E04014. https://doi.org/10. 1029/2004JE002245

Goldreich P (1965) Inclination of satellite orbits about an oblate precessing planet. Astron J 70:5-9

Greeley R (1987) Release of juvenile water on Mars: Estimated amounts and timing associated with volcanism. Science 236:1653-1654. https:// doi.org/10.1126/science.236.4809.1653

Grotzinger JP, Sumner DY, Kah LC, Stack K, Gupta S, Edgar L, Rubin D, Lewis K, Schieber J, Mangold N, Milliken R, Conrad PG, DesMarais D, Farmer J, Siebach K, Calef F, Hurowitz J, McLennan SM, Ming D, Vaniman D, Crisp J, Vasavada A, Edgett KS, Malin M, Blake D, Gellert R, Mahaffy P, Wiens RC, Maurice S, Grant JA, Wilson S, Anderson RC, Beegle L, Arvidson R, Hallet B, Sletten RS, Rice M, Bell J, Griffes J, Ehlmann B, Anderson RB, Bristow TF, Dietrich WE, Dromart G, Eigenbrode J, Fraeman A, Hardgrove C, Herkenhoff K, Jandura L, Kocurek G, Lee S, Leshin LA, Leveille R, Limonadi D, Maki J, McCloskey S, Meyer M, Minitti M, Newsom H, Oehler D, Okon A, Palucis M, Parker T, Rowland S, Schmidt M, Squyres S, Steele A, Stolper E, Summons R, Treiman A, Williams R, Yingst A (2014) A habitable fluvio-lacustrine environment at Yellowknife Bay, Gale Crater, Mars. Science. https://doi.org/10. 1126/science.1242777

Grün E, Zook HA, Fechtig H, Giese RH (1985) Collisional balance of the meteoritic complex. Icarus 62:244-272. https://doi.org/10.1016/ 0019-1035(85)90121-6

Gundlach B, Blum J (2013) A new method to determine the grain size of planetary regolith. Icarus 223:479-492. https://doi.org/10.1016/j. icarus.2012.11.039

Hartmann WK, Ryder G, Dones L, Grinspoon D (2000) Origin of the Earth and Moon. The University of Arizona Press, Tucson and Lunar Planetary Institute, Houston

Heavens NG, Kleinböhl A, Chaffin MS, Halekas JS, Kass DM, Hayne PO, McCleese DJ, Piqueux S, Shirley JH, Schofield JT (2018) Hydrogen escape from Mars enhanced by deep convection in dust storms. Nat Astron 2:126-132. https://doi.org/10.1038/s41550-017-0353-4 
Hemmi R, Miyamoto H (2020) Morphology and Morphometry of Sub-kilometer Craters on the Nearside of Phobos and Implications for Regolith Properties. Trans Jpn Soc Aeronaut Space Sci 63:124-131. https://doi. org/10.2322/tjsass.63.124

Hesselbrock AJ, Minton DA (2017) An ongoing satellite-ring cycle of Mars and the origins of Phobos and Deimos. Nat Geosci 10:266-269. https://doi.org/10.1038/ngeo2916

Hirata N, Barnouin-Jha OS, Honda C, Nakamura R, Miyamoto H, Sasaki S, Demura H, Nakamura AM, Michikami T, Gaskell RW, Saito J (2009) A survey of possible impact structures on 25143 Itokawa. Icarus 200:486-502. https://doi.org/10.1016/j.icarus.2008.10.027

Hunten DM (1979) Capture of Phobos and Deimos by protoatmospheric drag. Icarus 37:113-123. https://doi.org/10.1016/0019-1035(79) 90119-2

Hyodo R, Usui T (2021) Searching for life on Mars and its moons. Science 373:742. https://doi.org/10.1126/science.abj1512

Hyodo R, Genda H, Charnoz S, Pignatale FC, Rosenblatt P (2018) On the impact origin of Phobos and Deimos. IV Volatile Depletion. Astrophys J 860:150. https://doi.org/10.3847/1538-4357/aac024

Hyodo R, Kurosawa K, Genda H, Usui T, Fujita K (2019) Transport of impact ejecta from Mars to its moons as a means to reveal Martian history. Sci Rep 9:19833. https://doi.org/10.1038/s41598-019-56139-x

Hyodo R, Genda H, Charnoz S, Rosenblatt P (2017) On the impact origin of Phobos and Deimos. I. Thermodynamic and physical aspects. Astrophys J 845:125. https://doi.org/10.3847/1538-4357/aa81c4

Jacobson RA (2010) The orbits and masses of the Martian satellites and the libration of Phobos. Astron J 139:668-679. https://doi.org/10.1088/ 0004-6256/139/2/668

Jakosky BM, Pepin RO, Johnson RE, Fox JL (1994) Mars atmospheric loss and isotopic fractionation by solar-wind-induced sputtering and photochemical escape. Icarus 111:271-288. https://doi.org/10.1006/ icar.1994.1145

Jakosky BM, Brain D, Chaffin M, Curry S, Grebowsky J, Grebowsky J, Halekas J, Leblanc F, Lillis R, Luhmann JG, Andersson L, Andre N, Andrews D, Baird D, Baker D, Bell J, Benna M, Bhattacharyya D, Bougher S, Bowers C, Chamberlin P, Chaufray J-Y, Clarke J, Collinson G, Combi M, Connerney J, Connour K, Correira J, Crabb K, Crary F, Cravens T, Crismani M, Delory G, Dewey R, DiBraccio G, Dong C, Dong Y, Dunn P, Egan H, Elrod M, England S, Eparvier F, Ergun R, Eriksson A, Esman T, Espley J, Evans S, Fallows K, Fang X, Fillingim M, Flynn C, Fogle A, Fowler C, Fox J, Fujimoto M, Garnier P, Girazian Z, Groeller H, Gruesbeck J, Hamil O, Hanley KG, Hara T, Harada Y, Hermann J, Holmberg M, Holsclaw G, Houston S, Inui S, Jain S, Jolitz R, Kotova A, Kuroda T, Larson D, Lee Y, Lee C, Lefevre F, Lentz C, Lo D, Lugo R, Ma Y-J, Mahaffy P, Marquette ML, Matsumoto Y, Mayyasi M, Mazelle C, McClintock W, McFadden J, Medvedev A, Mendillo M, Meziane K, Milby Z, Mitchell D, Modolo R, Montmessin F, Nagy A, Nakagawa H, Narvaez C, Olsen K, Pawlowski D, Peterson W, Rahmati A, Roeten K, Romanelli N, Ruhunusiri S, Russell C, Sakai S, Schneider N, Seki K, Sharrar R, Shaver S, Siskind DE, Slipski M, Soobiah Y, Steckiewicz M, Stevens MH, Stewart I, Stiepen A, Stone S, Tenishev V, Terada N, Terada K, Thiemann E, Tolson R, Toth G, Trovato J, Vogt M, Weber T, Withers P, Xu S, Yelle R, Yiğit E, Zurek $\mathrm{R}$ (2018) Loss of the Martian atmosphere to space: present-day loss rates determined from MAVEN observations and integrated loss through time. Icarus 315:146-157. https://doi.org/10.1016/j.icarus. 2018.05.030

JAXA Hayabusa2 Project (2021) Asteroid explorer, Hayabusa2, reporter briefing, March 19, 2021, https://www.hayabusa2.jaxa.jp/en/enjoy/ material/

Jourdan F, Timms NE, Eroglu E, Mayers C, Frew A, Bland PA, Collins GS, Davison TM, Abe M, Yada T (2017) Collisional history of asteroid Itokawa. Geology 45:819-822. https://doi.org/10.1130/G39138.1

Kallemeyn GW, Wasson JT (1981) The compositional classification of chondrites-I. The carbonaceous chondrite groups. Geochim Cosmochim Acta 45:1217-1230. https://doi.org/10.1016/0016-7037(81)90145-9

Kameda S, Ozaki M, Enya K, Fuse R, Kouyama T, Sakatani N, Suzuki H, Osada N, Kato H, Miyamoto H, Yamazaki A, Nakamura T, Okamoto T, Ishimaru T, Hong P, Ishibashi K, Takashima T, Ishigami R, Kuo CL, Abe S, Goda Y, Murao H, Fujishima S, Aoyama T, Hagiwara K, Mizumoto S, Tanaka N, Murakami K, Matsumoto M, Tanaka K, Sakuta H (2021)
Design of Telescopic Nadir Imager for Geomorphology (TENGOO) and Observation of surface Reflectance by Optical Chromatic Imager (OROCHI) for the MMX mission. Earth Planets Space. https://doi.org/ 10.1186/s40623-021-01462-9

Karachevtseva IP, Oberst J, Zubarev AE, Nadezhdina IE, Kokhanov AA, Garov AS, Uchaev DV, DmV U, Malinnikov VA, Klimkin ND (2014) The Phobos information system. Planet Space Sci 102:74-85. https://doi.org/10. 1016/j.pss.2013.12.015

Kawakatsu Y, Kuramoto K, Ogawa N, Ikeda H, Ono G, Sawada H, Imada T, Otsuki M, Otake H, Muller R, Zacny K, Satoh Y, Yamada K, Mary S, Grebenstein M, Yoshikawa K (2019) Mission definition of Martian Moons eXploration (MMX). In: 70th international astronautical congress. IAC-19, A3, 4B, 7, x51465.

Kilgore TR, Burns JA, Pollack JB (1978) Orbital evolution of "Phobos" following its "capture." Bull Am Astron Soc 10:593

Kita NT, Yin QZ, MacPherson GJ, Ushikubo T, Jacobsen B, Nagashima K, Kurahashi E, Krot AN, Jacobsen SB (2013) ${ }^{26} \mathrm{Al}-{ }^{26} \mathrm{Mg}$ isotope systematics of the first solids in the early solar system. Meteorit Planet Sci 48:1383-1400. https://doi.org/10.1111/maps.12141

Kiuchi M, Nakamura AM (2014) Relationship between regolith particle size and porosity on small bodies. Icarus 239:291-293. https://doi.org/10. 1016/j.icarus.2014.05.029

Kleine T, Touboul M, Bourdon B, Nimmo F, Mezger K, Palme H, Jacobsen SB, Yin QZ, Halliday AN (2009) Hf-W chronology of the accretion and early evolution of asteroids and terrestrial planets. Geochim Cosmochim Acta 73:5150-5188. https://doi.org/10.1016/j.gca.2008.11.047

Kobayashi M, Krüger H, Senshu H, Wada K, Okudaira O, Sasaki S, Kimura H (2018) In situ observations of dust particles in Martian dust belts using a large-sensitive-area dust sensor. Planet Space Sci 156:41-46. https://doi.org/10.1016/j.pss.2017.12.011

Kokubo E, Ida S (1998) Oligarchic growth of protoplanets. Icarus 131:171178. https://doi.org/10.1006/icar.1997.5840

Krivov AV, Hamilton DP (1997) Martian dust belts: waiting for discovery. Icarus 128:335-353. https://doi.org/10.1006/icar.1997.5753

Lawrence DJ, Peplowski PN, Beck AW, Burks MT, Chabot NL, Cully MJ, Elphic RC, Ernst CM, Fix S, Goldsten JO, Hoffer EM, Kusano H, Murchie SL, Schratz BC, Usui T, Yokley ZW (2019) Measuring the Elemental Composition of Phobos: the Mars-moon Exploration with GAmma rays and NEutrons (MEGANE) Investigation for the Martian Moons eXploration (MMX) mission. Earth and Space Science 6:2605-2623. https://doi.org/10.1029/2019EA000811

Leshin LA, Mahaffy PR, Webster CR, Cabane M, Coll P, Conrad PG, Archer PD, Atreya SK, Brunner AE, Buch A, Eigenbrode JL (2013) Volatile, isotope, and organic analysis of martian fines with the Mars Curiosity rover. Science 341:1238937. https://doi.org/10.1126/science.1238937

Mahaffy PR, Webster CR, Atreya SK, Franz H, Wong M, Conrad PG, Harpold D, Jones JJ, Leshin LA, Manning H, Owen T, Pepin RO, Squyres S, Trainer M, MSL Science Team (2013) Abundance and isotopic composition of gases in the Martian atmosphere from the Curiosity rover. Science 341:263-266. https://doi.org/10.1126/science.1237966

Matsumoto K, Ikeda H (2016) Inhomogeneous two-layer internal structure and moments of inertia of Phobos. In: Lunar and planetary science conference (No. 1903, p. 1846).

Matsumoto K, Hirata N, Ikeda H, Kouyama T, Senshu H, Yamamoto K, Noda H, Miyamoto H, Araya A, Araki H, Kamata S, Baresi N, Namiki N(2021) MMX geodesy investigations: science requirements and observation strategy. Earth Planets Space. https://doi.org/10.1186/ s40623-021-01500-6

McSween HY Jr (2015) Petrology on Mars. Am Miner 100:2380-2395. https://doi.org/10.2138/am-2015-5257

Melchiorri R, Encrenaz T, Fouchet T, Drossart P, Lellouch E, Gondet B, Bibring JP, Langevin Y, Schmitt B, Titov D, lgnatiev N (2007) Water vapor mapping on Mars using OMEGA/Mars Express. Planet Space Sci 55:333-342. https://doi.org/10.1016/j.pss.2006.05.040

Michel P, Ballouz R-L, Barnouin OS, Jutzi M, Walsh KJ, May BH, Manzoni C, Richardson DC, Schwartz SR, Sugita S, Watanabe S, Miyamoto H, Hirabayashi M, Bottke WF, Connolly HC Jr, Yoshikawa M, Lauretta DS (2020) Collisional formation of top-shaped asteroids and implications for the origins of Ryugu and Bennu. Nat Commun 11:2665. https:// doi.org/10.1038/s41467-020-16433-z 
Michel P, Ulamec S, Boettger U, Grott M, Murdoch N, Vernazza P, Sunday C, Zhang Y, Valette R, Castellani R, Biele J, Tardivel S, Groussin O, Jorda L, Knollenberg J, Grundmann JT, Arrat D, Pont G, Mary S, Grebenstein M, Miyamoto H, Nakamura T, Wada K, Yoshikawa K, Kuramoto K(2021) The MMX rover: performing in-situ surface investigations on Phobos. Earth Planets Space. https://doi.org/10.1186/s40623-021-01464-7

Miyamoto H, Niihara T, Wada K, Ogawa K, Senshu H, Michel P, Kikuchi H, Hemmi R, Nakamura T, Nakamura AM, Hirata N, Sasaki S, Asphaug E, Britt DT, Abell PA, Ballouz RL, Banouin OS, Baresi N, Barucci MA, Biele J, Grott M, Hino H, Hong PK, Kameda S, Kobayashi M, Libourel G, Mogi K, Murdoch N, Nishio Y, Okamoto S, Ota Y, Otsuki M, Otto KA, Sakatani N, Shimizu Y, Takemura T, Terada N, Tsukamoto M, Usui T, Konrad W (2021) Surface environment of phobos and phobos simulant UPTS. Earth Planets Space. https://doi.org/10.1186/ s40623-021-01406-3

Mumma MJ, Charnley SB (2011) The chemical composition of cometsemerging taxonomies and natal heritage. Ann Rev Astron Astrophys 49:471-524. https://doi.org/10.1146/annurev-astro-081309-130811

Murray JB, Heggie DC (2014) Character and origin of Phobos' grooves. Planet Space Sci 102:119-143. https://doi.org/10.1016/j.pss.2014.03. 001

Murchie SL, Thomas PC, Rivkin AS, Chabot NL (2015). Phobos and Deimos. In: Michel P, DeMeo FE, Bottke WF (eds.) Asteroids IV, University of Arizona Press, Tucson, 895 pp. p.451-467. Doi: https://doi.org/10. 2458/azu_uapress_9780816532131-ch024

Murray JB, Rothery DA, Thornhill GD, Muller JP, lliffe JC, Day T, Cook AC (1994) The origin of Phobos' grooves and crater chains. Planet Space Sci 42:519-526. https://doi.org/10.1016/0032-0633(94)90093-0

Mustard JF, Cooper CD, Rifkin MK (2001) Evidence for recent climate change on Mars from the identification of youthful near-surface ground ice. Nature 412:411-414. https://doi.org/10.1038/35086515

Nagao K, Okazaki R, Nakamura T, Miura YN, Osawa T, Bajo K, Matsuda S, Ebihara M, Ireland TR, Kitajima F, Naraoka H, Noguchi T, Tsuchiyama A, Yurimoto H, Zolensky ME, Uesugi M, Shirai K, Abe M, Yada T, Ishibashi Y, Fujimura A, Mukai T, Ueno M, Okada T, Yoshikawa M, Kawaguchi $J$ (2011) Irradiation history of Itokawa regolith material deduced from noble gases in the Hayabusa samples. Science 333:1128-1131. https://doi.org/10.1126/science.1207785

Nakamura T, Noguchi T, Tanaka M, Zolensky ME, Kimura M, Tsuchiyama A, Nakato A, Ogami T, Ishida H, Uesugi M, Yada T, Shirai K, Fujimura A, Okazaki R, Sandford SA, Ishibashi Y, Abe M, Okada T, Ueno M, Mukai T, Yoshikawa M, Kawaguchi J (2011) Itokawa dust particles: a direct link between S-type asteroids and ordinary chondrites. Science 333:1113-1116. https://doi.org/10.1126/science.1207758

Nakamura T, Ikeda H, Kouyama T, Nakagawa H, Kusano H, Senshu H, Kameda S, Matsumoto K, Gonzalez-Franquesa F, Ozaki N, Takeo Y, Baresi N, Oki Y, Lawrence DJ, Chabot NL, Peplowski PN, Barucci MA, Sawyer E, Yokota S, Terada N, Ulamec S, Michel P, Kobayashi M, Sasaki S, Hirata N, Wada K, Miyamoto H, Imamura T, Ogawa N, Ogawa K, Iwata T, Imada T, Otake H, Canalias E, Lorda L, Tardivel S, Mary S, Kunugi M, Mitsuhashi S, Doressoundiram A, Merlin F, Fornasier S, Reess JM, Bernardi P, Imai S, Ito Y, Ishida H, Kuramoto K, Kawakatsu Y (2021) Science operation plan of Phobos and Deimos from the MMX spacecraft. Earth Planets Space. https://doi.org/10.1186/ s40623-021-01546-6

Nakashima D, Kita NT, Ushikubo T, Noguchi T, Nakamura T, Valley JW (2013) Oxygen three-isotope ratios of silicate particles returned from asteroid Itokawa by the Hayabusa spacecraft: a strong link with equilibrated LL chondrites. Earth Planet Sci Lett 379:127-136. https:// doi.org/10.1016/j.epsl.2013.08.009

Nénon Q, Poppe AR, Rahmati A, Lee CO, McFadden JP, Fowler CM (2019) Phobos surface sputtering as inferred from MAVEN ion observations. J Geophys Res 124:3385-3401. https://doi.org/10.1029/2019JE0061 97

Nénon Q, Poppe AR, Rahmati A, McFadden JP (2021) Implantation of Martian atmospheric ions within the regolith of Phobos. Nat Geosci 14:61-66. https://doi.org/10.1038/s41561-020-00682-0

Nishiizumi K, Arnold JR, Kohl CP, Caffee MW, Masarik J, Reedy RC (2009) Solar cosmic ray records in lunar rock 64455. Geochim Cosmochim Acta 73:2163-2176. https://doi.org/10.1016/.j.gca.2008.12.021
Ogohara K, Nakagawa H, Aoki S, Kouyama T, Usui T, Terada N, Imamura T, Montmessin F, Brain D, Doressoundiram A, Gautier T, Hara T, Harada Y, Ikeda H, Koike M, Leblanc F, Ramirez R, Sawyer E, Seki K, Spiga A, Vandaele AC, Yokota S, Barucci A, Kameda S(2021) The Mars system revealed by the Martian Moons eXploration mission. Earth Planets Space. https://doi.org/10.1186/s40623-021-01417-0

Pang KD, Pollack JB, Veverka J, Lane AL, Ajello JM (1978) The composition of Phobos: evidence for carbonaceous chondrite surface from spectral analysis. Science 199:64-66. https://doi.org/10.1126/science.199. 4324.64

Pang KD, Rhoads JW, Lane AL, Ajello JM (1980) Spectral evidence for a carbonaceous chondrite surface composition on Deimos. Nature 283:277-278. https://doi.org/10.1038/283277a0

Park J, Turrin BD, Herzog GF, Lindsay FN, Delaney JS, Swisher CC III, Uesugi M, Karouji Y, Yada T, Abe M, Okada T, Ishibashi Y $(2015){ }^{40} \mathrm{Ar} /{ }^{39} \mathrm{Ar}$ age of material returned from asteroid 25143 Itokawa. Meteorit Planet Sci 50:2087-2098. https://doi.org/10.1111/maps.12564

Pollack JB, Burns JA, Tauber ME (1979a) Gas drag in primordial circumplanetary envelopes: a mechanism for satellite capture. Icarus 37:587-611. https://doi.org/10.1016/0019-1035(79)90016-2

Pollack JB, Colburn DS, Flasar FM, Kahn R, Carlston CE, Pidek D (1979b) Properties and effects of dust particles suspended in the Martian atmosphere. J Geophys Res Solid Earth 84:2929-2945. https://doi. org/10.1029/JB084iB06p02929

Poppe AR, Curry SM (2014) Martian planetary heavy ion sputtering of Phobos. Geophys Res Lett 41:6335-6341. https://doi.org/10.1002/ 2014GL061100

Poppe AR, Curry SM, Fatemi S (2016) The Phobos neutral and ionized torus. J Geophys Res 121:770-783. https://doi.org/10.1002/2015JE004948

Ramsley KR, Head JW (2013) Mars impact ejecta in the regolith of Phobos: bulk concentration and distribution. Planet Space Sci 87:115-129. https://doi.org/10.1016/j.pss.2013.09.005

Ramstad R, Barabash S, Futaana Y, Nilsson H, Holmström M (2017) Global mars-solar wind coupling and ion escape. J Geophys Res 122:80518062. https://doi.org/10.1002/2017JA024306

Richardson JE Jr, Melosh HJ, Greenberg RJ, O'Brien DP (2005) The global effects of impact-induced seismic activity on fractured asteroid surface morphology. Icarus 179:325-349. https://doi.org/10.1016/j. icarus.2005.07.005

Rivkin AS, Brown RH, Trilling DE, Bell lii JF, Plassmann JH (2002) Near-infrared spectrophotometry of Phobos and Deimos. Icarus 156:64-75. https:// doi.org/10.1006/icar.2001.6767

Rosenblatt P (2011) The origin of the Martian moons revisited. Astron Astrophys Rev 19:44. https://doi.org/10.1007/s00159-011-0044-6

Rosenblatt P, Charnoz S, Dunseath KM, Terao-Dunseath M, Trinh A, Hyodo $\mathrm{R}$, Genda H, Toupin S (2016) Accretion of Phobos and Deimos in an extended debris disc stirred by transient moons. Nat Geosci 9:581-583. https://doi.org/10.1038/ngeo2742

Sawada H, Okazaki R, Tachibana S, Sakamoto K, Takano Y, Okamoto C, Yano H, Miura Y, Abe M, Hasegawa S, Noguchi T, Hayabusa2 SMP Team (2017) Hayabusa2 sampler: collection of asteroidal surface material. Space Sci Rev 208:81-106. https://doi.org/10.1007/ s11214-017-0338-8

Schaible MJ, Dukes CA, Hutcherson AC, Lee P, Collier MR, Johnson RE (2017) Solar wind sputtering rates of small bodies and ion mass spectrometry detection of secondary ions. J Geophys Res Planets 122:19681983. https://doi.org/10.1002/2017JE005359

Schmedemann N, Michael GG, Ivanov BA, Murray JB, Neukum G (2014) The age of Phobos and its largest crater, Stickney. Planet Space Sci 102:152-163. https://doi.org/10.1016/.jpss.2014.04.009

Senshu H, Mizuno T, Umetani K, Nakura T, Konishi A, Ogawa A, Ikeda H, Matsumoto K, Noda H, Ishihara Y, Sasaki S, Tateno N, Ikuse Y, Mayuzumi K, Kase T, Kashine H (2021) Light Detection and Ranging (LIDAR) laser altimeter for the Martian Moons eXploration (MMX) spacecraft. Earth Planets Space. https://doi.org/10.1186/s40623-021-01537-7

Soter S (1971) The dust belts of Mars. Report of Center for Radiophysics and Space Research, 462.

Spiga A, Faure J, Madeleine JB, Määttänen A, Forget F (2013) Rocket dust storms and detached dust layers in the Martian atmosphere. J Geophys Res Planets 118:746-767. https://doi.org/10.1002/jgre.20046 
Stöffler D, Keil K, Scott ERD (1991) Shock metamorphism of ordinary chondrites. Geochim Cosmochim Acta 55:3845-3867. https://doi.org/10 1016/0016-7037(91)90078-J

Sugita S, Honda R, Morota T, Kameda S, Sawada H, Tatsumi E, Yamada M, Honda C, Yokota Y, Kouyama T, Sakatani N, Ogawa K, Suzuki H, Okada T, Namiki N, Tanaka S, lijima Y, Yoshioka K, Hayakawa M, Cho Y, Matsuoka M, Hirata N, Hirata N, Miyamoto H, Domingue D, Hirabayashi M, Nakamura T, Hiroi T, Michikami T, Michel P, Ballouz R-L, Barnouin OS, Ernst CM, Schröder SE, Kikuchi H, Hemmi R, Komatsu G, Fukuhara T, Taguchi M, Arai T, Senshu H, Demura H, Ogawa Y, Shimaki Y, Sekiguchi T, Müller TG, Hagermann A, Mizuno T, Noda H, Matsumoto K, Yamada R, Ishihara Y, Ikeda H, Araki H, Yamamoto K, Abe S, Yoshida F, Higuchi A, Sasaki S, Oshigami S, Tsuruta S, Asari K, Tazawa S, Shizugami M, Kimura J, Otsubo T, Yabuta H, Hasegawa S, Ishiguro M, Tachibana S, Palmer E, Gaskell R, Le Corre L, Jaumann R, Otto K, Schmitz N, Abell PA, Barucci MA, Zolensky ME, Vilas F, Thuillet F, Sugimoto C, Takaki N, Suzuki Y, Kamiyoshihara H, Okada M, Nagata K, Fujimoto M, Yoshikawa M, Yamamoto Y, Shirai K, Noguchi R, Ogawa N, Terui F, Kikuchi S, Yamaguchi T, Oki Y, Takao Y, Takeuchi H, Ono G, Mimasu Y, Yoshikawa K, Takahashi T, Takei Y, Fujii A, Hirose C, Nakazawa S, Hosoda S, Mori O, Shimada T, Soldini S, Iwata T, Abe M, Yano H, Tsukizaki R, Ozaki M, Nishiyama K, Saiki T, Watanabe S, Tsuda Y (2019) The geomorphology, color, and thermal properties of Ryugu: implications for parent-body processes. Science 364:422. https://doi. org/10.1126/science.aaw0422

Szeto AM (1983) Orbital evolution and origin of the Martian satellites. Icarus 55:133-168. https://doi.org/10.1016/0019-1035(83)90056-8

Takemura T, Miyamoto H, Hemmi R, Niihara T, Michel P (2021) Small-scale topographic irregularities on Phobos: image and numerical analyses for MMX mission. Earth Planets Space. https://doi.org/10.1186/ s40623-021-01463-8

Terada K, Sano Y, Takahata N, Tsuchiyama A, Nakamura T, Noguchi T, Karouji Y, Uesugi M, Yada T, Nakabayashi M, Fukuda K, Nagahara H (2018) Thermal and impact histories of 25143 Itokawa recorded in Hayabusa particles. Sci Rep 8:11806. https://doi.org/10.1038/ s41598-018-30192-4

Thomas P (1979) Surface features of Phobos and Deimos. Icarus 40:223-243. https://doi.org/10.1016/0019-1035(79)90069-1

Thomas PC (1989) The shapes of small satellites. Icarus 77:248-274. https:// doi.org/10.1016/0019-1035(89)90089-4

Thomas PC (1993) Gravity, tides, and topography on small satellites and asteroids: application to surface features of the Martian satellites. Icarus 105:326-344. https://doi.org/10.1006/icar.1993.1130

Thomas PC, Adinolfi D, Helfenstein P, Simonelli D, Veverka J (1996) The surface of Deimos: contribution of materials and processes to its unique appearance. Icarus 123:536-556. https://doi.org/10.1006/icar. 1996.0177

Tolson RH, Duxbury TC, Born GH, Christensen EJ, Diehl RE, Farless D, Hildebrand CE, Mitchell RT, Molko PM, Morabito LA, Palluconi FD, Reichert RJ, Taraji H, Veverka J, Neugebauer G, Findlay JT (1978) Viking first encounter of Phobos: preliminary results. Science 199:61-64. https:// doi.org/10.1126/science.199.4324.61

Trinquier A, Birck JL, Allègre CJ, Göpel C, Ulfbeck D $(2008)^{53} \mathrm{Mn}-{ }^{53} \mathrm{Cr}$ systematics of the early Solar System revisited. Geochim Cosmochim Acta 72(20):5146-5163. https://doi.org/10.1016/j.gca.2008.03.023

Trotignon JG, Grard R, Barabash S, Lundin R, Dubinin E (1996) Solar wind measurements near Mars and their implication in the Red Planet environment. Planet Space Sci 44:117-127. https://doi.org/10.1016/ 0032-0633(95)00071-2

Tsuda Y, Yoshikawa M, Abe M, Minamino H, Nakazawa S (2013) System design of the Hayabusa 2-Asteroid sample return mission to 1999 JU3. Acta Astronaut 91:356-362. https://doi.org/10.1016/j.actaastro. 2013.06.028

Usui T, Alexander CMD, Wang J, Simon Jl, Jones JH (2015) Meteoritic evidence for a previously unrecognized hydrogen reservoir on Mars. Earth Planet Sci Lett 410:140-151. https://doi.org/10.1016/j.epsl. 2014.11.022

Usui T, Bajo KI, Fujiya W, Furukawa Y, Koike M, Miura YN, Sugawara H, Tachibana S, Takano Y, Kuramoto K (2020) The importance of Phobos sample return for understanding the Mars-moon system. Space Sci Rev 216:49. https://doi.org/10.1007/s11214-020-00668-9
Veverka J, Burns JA (1980) The moons of Mars. Annu Rev Earth Planet Sci 8:527-558. https://doi.org/10.1146/annurev.ea.08.050180.002523

Veverka J, Duxbury TC (1977) Viking observations of Phobos and Deimos: preliminary results. J Geophys Res 82:4213-4223. https://doi.org/10. 1029/JS082i028p04213

Villanueva GL, Mumma MJ, Novak RE, Käufl HU, Hartogh P, Encrenaz T, Tokunaga A, Khayat A, Smith MD (2015) Strong water isotopic anomalies in the Martian atmosphere: probing current and ancient reservoirs. Science 348:218-221. https://doi.org/10.1126/science.aaa3630

Walsh KJ, Morbidelli A, Raymond SN, O'Brien DP, Mandell AM (2011) A low mass for Mars from Jupiter's early gas-driven migration. Nature 475:206-209. https://doi.org/10.1038/nature10201

Warren PH (2011) Stable-isotopic anomalies and the accretionary assemblage of the Earth and Mars: a subordinate role for carbonaceous chondrites. Earth Planet Sci Lett 311:93-100. https://doi.org/10. 1016/j.epsl.2011.08.047

Wasson JT, Kallemeyn GW (1988) Compositions of chondrites. Philos Trans R Soc Lond Ser Math Phys Sci 325:535-544. https://doi.org/10.1098/ rsta. 1988.0066

Watanabe S, Hirabayashi M, Hirata N, Hirata N, Noguchi R, Shimaki Y, Ikeda H, Tatsumi E, Yoshikawa M, Kikuchi S, Yabuta H, Nakamura T, Tachibana S, Ishihara Y, Morota T, Kitazato K, Sakatani N, Matsumoto K, Wada K, Senshu H, Honda C, Michikami T, Takeuchi H, Kouyama T, Honda R, Kameda S, Fuse T, Miyamoto H, Komatsu G, Sugita S, Okada T, Namiki N, Arakawa M, Ishiguro M, Abe M, Gaskell R, Palmer E, Barnouin OS, Michel P, French AS, MCMahon JW, Scheeres DJ, Abell PA, Yamamoto Y, Tanaka S, Shirai K, Matsuoka M, Yamada M, Yokota Y, Suzuki H, Yoshioka K, Cho Y, Tanaka S, Nishikawa N, Sugiyama T, Kikuchi H, Hemmi R, Yamaguchi T, Ogawa N, Ono G, Mimasu Y, Yoshikawa K, Takahashi T, Takei Y, Fujii A, Hirose C, Iwata T, Hayakawa M, Hosoda S, Mori O, Sawada H, Shimada T, Soldini S, Yano H, Tsukizaki R, Ozaki M, lijima Y, Ogawa K, Fujimoto M, Ho T-M, Moussi A, Jaumann R, Bibring J-P, Krause C, Terui F, Saiki T, Nakazawa S, Tsuda Y (2019) Hayabusa2 arrives at the carbonaceous asteroid 162173 Ryugu-a spinning top-shaped rubble pile. Science 364:268-272. https://doi. org/10.1126/science.aav8032

Yabuta H, Noguchi T, Itoh S, Nakamura T, Miyake A, Tsujimoto S, Ohashi N, Sakamoto N, Hashiguchi M, Abe K, Okuboh A, David Kilcoyne AL, Tachibana S, Okazaki R, Terada K, Ebihara M, Nagahara H (2017) Formation of an ultracarbonaceous Antarctic micrometeorite through minimal aqueous alteration in a small porous icy body. Geochim Cosmochim Acta 214:172-190. https://doi.org/10.1016/j.gca.2017. 06.047

Yamamoto K, Yoshikawa M, Zolensky ME (2011) Oxygen isotopic compositions of asteroidal materials returned from Itokawa by the Hayabusa mission. Science 333:1116-1119. https://doi.org/10.1126/science. 1207776

Yoder CF (1982) Tidal rigidity of Phobos. Icarus 49:327-346. https://doi.org/ 10.1016/0019-1035(82)90040-9

Yokota S, Saito Y, Asamura K, Tanaka T, Nishino MN, Tsunakawa H, Shibuya H, Matsushima M, Shimizu H, Takahashi F, Fujimoto M, Mukai T, Terasawa T (2009) First direct detection of ions originating from the Moon by MAP-PACE IMA onboard SELENE (KAGUYA). Geophys Res Lett. https:// doi.org/10.1029/2009GL038185

Yokota S, Terada N, Matsuoka A, Murata N, Saito Y, Delcourt D, Futaana Y, Seki K, Schaible MJ, Asamura K, Kasahara S, Nakagawa H, Nishino MN, Nomura R, Keika K, Harada Y, Imajo S (2021) In situ observations of ions and magnetic field around Phobos: The Mass Spectrum Analyzer (MSA) for the Martian Moons eXploration (MMX) mission. Earth Planets Space. https://doi.org/10.1186/s40623-021-01452-x

Yoshizaki T, McDonough WF (2020) The composition of Mars. Geochim Cosmochim Acta 273:137-162. https://doi.org/10.1016/j.gca.2020.01.011

Yurimoto H, Abe K, Abe M, Ebihara M, Fujimura A, Hashiguchi M, Hashizume K, Ireland TR, Itoh S, Katayama J, Kato C, Kawaguchi J, Kawasaki N, Kitajima F, Kobayashi S, Meike T, Mukai T, Nagao K, Nakamura T, Naraoka H, Noguchi T, Okazaki R, Park C, Sakamoto N, Seto Y, Takei M, Tsuchiyama A, Uesugi M, Wakaki S, Yada T, Yamamoto K, Yoshikawa M, Zolensky ME (2011) Oxygen isotopic compositions of asteroidal materials returned from Itokawa by the Hayabusa mission. Science 333:1116-1119. https://doi.org/10.1126/science.1207776 
Zellner BH, Capen RC (1974) Photometric properties of the Martian satellites. Icarus 23:437-444. https://doi.org/10.1016/0019-1035(74) 90062-1

\section{Publisher's Note}

Springer Nature remains neutral with regard to jurisdictional claims in published maps and institutional affiliations.

Submit your manuscript to a SpringerOpen ${ }^{\circ}$ journal and benefit from:

- Convenient online submission

- Rigorous peer review

- Open access: articles freely available online

- High visibility within the field

- Retaining the copyright to your article

Submit your next manuscript at $\boldsymbol{\nabla}$ springeropen.com 